FINAL REPORT

on

NEER Grant Program \# DE-PS07-99ID13730

\title{
Advanced Algorithms and Automation Tools for Discrete Ordinates Methods in Parallel Environments
}

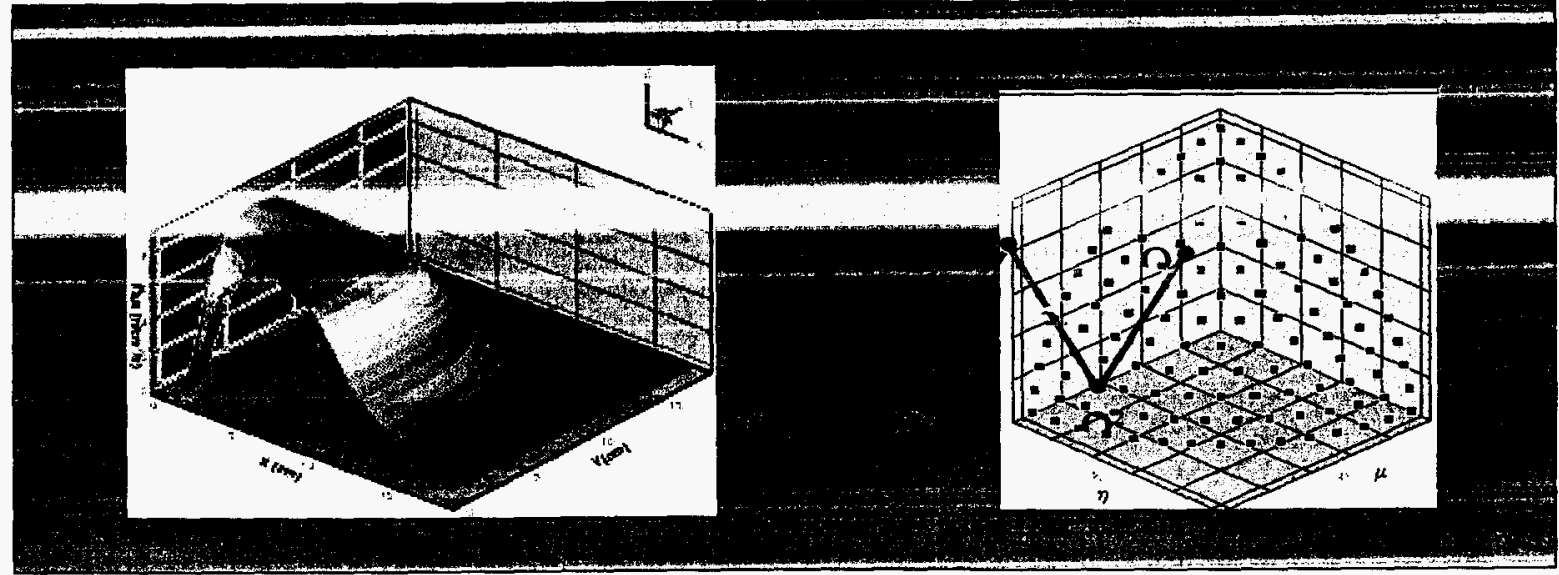

by

?

A. Haghighat

Nuclear and Radiological Engineering Department

202 Nuclear Sciences Building

University of Florida

Gainesville, FL 32611

Submitted to:

Mrs. Nancy Elizondo

US Department of Energy

Idaho Operation Office

850 Engineering Drive, MS 1216

Idaho Falls, ID 83401-1563

elizonna@id.doe.gov 


\section{Introduction}

This final report discusses major accomplishments of a 3-year project (\#DE-PS07-99ID13730) under the DOE's NEER Program. The project has developed innovative and automated algorithms, codes, and tools for solving the discrete ordinates particle transport method efficiently in parallel environments. Using a number of benchmark and real-life problems, the performance and accuracy of the new algorithms have been measured and analyzed.

The project has been performed by Prof. Haghighat and Dr. Sjoden and four graduate students in nuclear engineering, and resulted in 2 completed and 1 in-progress $\mathrm{PhD}$ dissertations, $1 \mathrm{MS}$ thesis, and 21 publications. Further, two of the publications received awards: i) "Development of the Regional Angular Refinement and its Application to the CT-Scan device," 2002 ANS Meeting, Hollywood, FL, June 2002, by G. Longoni and A. Haghighat; ii) "Development of An Expert System for Preparing an Effective Mesh Distribution for the Sn Method in the Parallel Environment," $11^{\text {th }}$ International Symposium on Reactor Dosimetry (ISRD), August 2002, by A. Patchimpattapong and A. Haghighat.

Over the past three years, following tasks have been completed:

i) Demonstration of the effectiveness of the adaptive differencing strategy in conjunction with the Taylor Projection Mesh Coupling (TPMC)

ii) Development of new multigrid algorithms for acceleration of solution convergence, and tested these algorithms for benchmark and real-life problems.

iii) Development of new schemes for generation of angular quadrature sets for being able to simulate highly angular dependent problems

iv) Development of a general 3-D parallel Simplified $\mathrm{P}_{\mathrm{L}}\left(\mathrm{SP}_{\mathrm{L}}\right)$ algorithm for acceleration of the $S_{N}$ method. (Utilized special numerical formulation for reducing the computational cost of the $\mathrm{SP}_{\mathrm{L}}$ formulation.)

v) Development of an acceleration algorithm which uses the $\mathrm{SP}_{\mathrm{L}}$ solution to increase the rate of convergence of the $S_{N}$ method.

vi) Development of an expert system for mesh generation and for selection of an appropriate domain decomposition algorithm in a parallel environment.

Chapters II-VII provide some discussions on each task, and refer to our publications which provide further detail on each task. Finally Chapter VIII provides an overall conclusion for this study. 


\section{II- Demonstration of the effectiveness of the adaptive differencing strategy in conjunction with the Taylor Projection Mesh Coupling (TPMC)}

\section{II.1 Introduction}

Numerous studies have been devoted to developing differencing schemes for $S_{N}$ method. These schemes may or may not possess desirable properties including positivity, accuracy, efficiency, and free of non-physical oscillations [Alcouffe and O'Dell, 1986; Alcouffe et al., 1979; Rhoades and Engle, 1977; Petrovic and Haghighat, 1996; Petrovic and Haghighat, 1998; Sjoden and Haghighat, 1997a; Sjoden, 1997]. Often a single differencing formulation cannot satisfy all the properties in all physical situations. Since most real-life problems are composed of different materials of various optical path lengths and dimensions, the use of a single differencing scheme may result in solution inaccuracy, or redundant meshing and computational inefficiency.

We have developed an adaptive differencing strategy (ADS) [Sjoden and Haghighat, 1996a], Ref.) which allows for variation of the differencing scheme throughout a physical model. Moreover, to conserve the computational time and memory, we have developed a projection formulation (i.e. Taylor Projection Mesh Coupling, TPMC [Sjoden and Haghighat, 1996b] for accurate projection of angular flux across regions with fine- and coarse-grid densities. These algorithms have been incorporated and tested within the PENTRAN (Parallel Environment Neutral-particle TRANsport) code which is a three-dimensional parallel discrete ordinates code designed for solving large and complex radiation transport problems in distributed-memory and distributed-computing environments [Sjoden and Haghighat, 1997b].

To examine the effectiveness of the new formulations, we use the Kobayashi 3-D simple benchmarks [Kobayashi, 1996]. The standard discrete ordinates method, with its limited number of directions, generally yields erroneous results that may differ from the true solutions by several orders of magnitude. This is especially true for deep penetration in a purely absorbing or low scattering shield, or at the interface of void and shield. With this study, we demonstrated that new formulations including variable meshing along all axes, ADS and TPMC can be very effective for solving such problems. Since the benchmarks had a simple one-group cross-section with $P_{0}$ anisotropy, we used the serial version of PENTRAN on a single processor of the Penn State LIONX PC-Cluster.

For the current problems, since we are interested in determining flux values at positions separated from the source by several mfp of a purely absorbing or low scattering material with void regions, the current ADS uses either DTW [Petrovic and Haghighat, 1998] or EDW [Sjoden and Haghighat, 1997a] schemes. For the most part, DTW is used in the void region and the 50\% scattering shield, and the EDW scheme is used in the pure absorber. To overcome the limited number of directions available in a level-symmetric quadrature set, we design a discrete model with a variable mesh arrangement that provides "smearing" of angular fluxes over the large interval meshes. To preserve solution accuracy as we increase the mesh size, the ADS and TPMC are used.

To estimate the accuracy, we compared PENTRAN (i.e., new formulations) fluxes to the analytical solutions in the pure absorber case, and to the Monte Carlo solutions in the 50\% scattering case [Kobayashi et al., 1999]. In this report, we only discuss the results obtained for 
the pure absorber cases. Haghighat and Sjoden, 1999c, and Haghighat et al., 2001 provide detailed discussions on both pure absorber and $50 \%$ scattering problems, and demonstrate the impact of the TPMC and ADS formulations on the solution accuracy. The reminder of this Chapter is allocated to discussions on the three Kobayashi benchmark problems and the comparison of PENTRAN solutions to the reference solutions provided in Kobayashi et al., 1999.

\section{I.1 PENTRAN MODELS AND SOLUTIONS FOR THE KOBAYASHI BENCHMARKS}

The three Kobayashi problems [Kobayashi, 1996] are parallelepiped or cubic in shape and contain three r egions: s ource, void, and s hield. Problems a re solved for two situations: $i$ ) the material in the source and shield regions is a pure absorber; ii) the material in the source and shield r egions has a $50 \%$ s cattering $r$ atio. The total c ross-section of this m aterial is $0.1 \mathrm{~cm}^{-1}$, while the total cross-section of the void is $10^{-4} \mathrm{~cm}^{-1}$.

To develop the PENTRAN models, we utilized the PENMSH [Haghighat, 1998] code for preparation of mesh, material and source distributions, and the PENINP [Haghighat, 1999a] code for automatic preparation of a PENTRAN input file. For post-processing, we used the PENDATA code [Sjoden, 1999] for preparation of tables of 3-D flux values, and the PENPRL code [Haghighat, 1999b] for extracting flux values at specific positions for which we have analytical or Monte Carlo solutions [Kobayashi et al., 1999].

All the calculations except when indicated are performed using an S20 level-symmetric quadrature set and a single processor of the Penn State PC-Cluster. This machine has an 1686 processor and 1 Gbyte of memory. As mentioned earlier, here we only show the results for the pure absorber case; the 50\% case results are presented in Haghighat et al., 2001.

PROBLEM 1 - SHIELD WITH SQUARE VOID

Figure 1 shows problem 1, referred to as the "shield with square void," is made of three cubic regions: source, void, and shield. The problem size is $100 \times 100 \times 100 \mathrm{~cm}^{3}$, and the source region is $10 \times 10 \times 10 \mathrm{~cm}^{3}$. The void region is between 10 and $50 \mathrm{~cm}$ along $x, y$, and $z$ axes.

The PENTRAN model is comprised of seven coarse z-levels. The axial widths of levels 1 to 7 are 10, 10, $20,10,10,20$, and $20 \mathrm{~cm}$, respectively. Each level is partitioned into 5 axial fine meshes. The $x-y$ planes are partitioned into $7 \times 7$ coarse meshes. These meshes are further refined, resulting in a total of 31550 meshes for the whole model. This same mesh distribution is used for both the pure absorber and the $50 \%$ scattering cases.

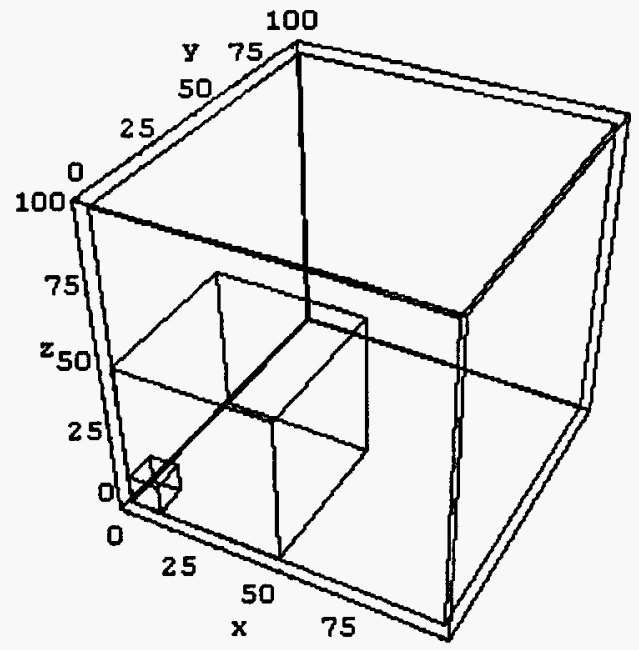

Figure 1 Kobayashi Benchmark Problem 1 ; shield with square void. 
PENTRAN results are compared to the analytical or Monte Carlo solutions for three sets of positions. First set (case 1A) includes positions along the y-axis for every $10 \mathrm{~cm}$ interval between $y=5 \mathrm{~cm}$ and $y=95 \mathrm{~cm}$, at $x=5 \mathrm{~cm}$ and $\mathrm{z}=5 \mathrm{~cm}$. The second set (case 1B) includes positions along the main diagonal $(x=y=z$ positions) of the physical model between points $(5,5,5)$ and $(95$, 95,95 ). The third set (case 1C) includes positions at $z=5 \mathrm{~cm}, y=65 \mathrm{~cm}$, and along the $x$-axis at $10 \mathrm{~cm}$ intervals between 5 and $95 \mathrm{~cm}$.

A PENTRAN calculation was performed for the purely absorbing shield in $\sim 207 \mathrm{sec}$. Figure 2 shows the 3-D flux distribution projected onto the 3-D mesh cells.

Comparison of the PENTRAN and analytical fluxes for the three sets of positions (cases 1A to 1C) indicate the following: for case $1 \mathrm{~A}$ positions, along the $y$-axis, the maximum difference is $<9 \%$; For case $1 \mathrm{~B}$ positions, along the main diagonal, the maximum difference of $<\sim 37 \%$ occurs at position of $(65,65,65)$ that is adjacent to the corner of the void region; For case $1 \mathrm{C}$, along the $\mathrm{x}$-axis, the maximum difference of $<27 \%$ occurs at $x=55 \mathrm{~cm}$ that is located adjacent to the outer corner of the void region.

\section{PROBLEM 2 - SHIELD WITH VOID DUCT}

Figure 3 shows problem 2 , referred to as the shield with a void duct, consisting of three regions: source, void duct, and shield. The problem size is $60 \times 100 \times 60 \mathrm{~cm}^{3}$, the source region is $10 \times 10 \times 10 \mathrm{~cm}^{3}$, and the void duct is along $y$-axis between $y=10$ and $100 \mathrm{~cm}, x=0.0$ and $10.0 \mathrm{~cm}$, and $\mathrm{z}=0.0$ and $10.0 \mathrm{~cm}$.

PENTRAN results are compared to the analytical solutions for two sets of positions. The first set (case $2 \mathrm{~A}$ ) includes positions along the $y$-axis at every $10 \mathrm{~cm}$ between 5 and $95 \mathrm{~cm}$, at $x=5 \mathrm{~cm}$ and $z=5 \mathrm{~cm}$. The second set (case 2B) includes positions along $\mathrm{x}$-axis at every 10 $\mathrm{cm}$ between 5 and $95 \mathrm{~cm}$, at $\mathrm{z}=5 \mathrm{~cm}$ and $\mathrm{y}=95 \mathrm{~cm}$.

The PENTRAN model has only one z-level of thickness $10 \mathrm{~cm}$, which is partitioned into 5 axial meshes. The $x-y$ plane is partitioned into $6 \times 10$ coarse meshes. These meshes are refined further into a total of 1617 meshes. Note that mesh refinement is done only along the diagonals between the source and positions of comparison. The PENTRAN calculation is completed in $17.3 \mathrm{sec}$. Figure 4 shows the 3-D flux distribution projected onto the 3-D mesh cells. Comparison of the PENTRAN and analytical fluxes for

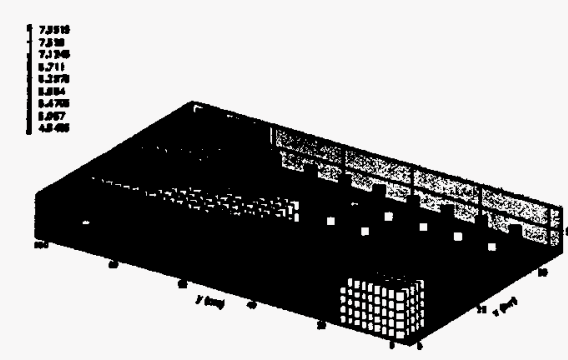

Figure 4 Flux distribution in Problem 2 with pure absorber. 
the two sets of positions (cases $2 \mathrm{~A}$ and $2 \mathrm{~B}$ ) indicates: For case $2 \mathrm{~A}$ positions, along the $\mathrm{y}$-axis, the maximum difference is $<8 \%$. For case $2 \mathrm{~B}$ positions, along the $\mathrm{x}$-axis, a maximum difference of $<\sim 24 \%$ occurs at $x=25 \mathrm{~cm}$.

\section{PROBLEM 3 - SHIELD WITH DOG-LEG VOID DUCT}

Figure 5 shows problem 3 , referred to as the shield with dog-leg void duct, composed of three regions: source, void ducts, and shield. Problem size is $60 \times 100 \times 60 \mathrm{~cm}^{3}$, source region is $10 \times 10 \times 10 \mathrm{~cm}^{3}$, and void duct penetrates through model.

PENTRAN results are compared to the analytical and Monte Carlo solutions for three sets of positions. The first set (case 3A) includes positions along the $y$-axis at every 10 $\mathrm{cm}$ between 5 and $95 \mathrm{~cm}$, at $x=5 \mathrm{~cm}$ and $z=5 \mathrm{~cm}$. The second set (case $3 \mathrm{~B}$ ) includes positions along $x$-axis at every $10 \mathrm{~cm}$ between 5 and $55 \mathrm{~cm}$, at $z=5 \mathrm{~cm}$ and $y=55 \mathrm{~cm}$. The third set (case $3 \mathrm{C}$ ) includes positions along $x$-axis at every 10

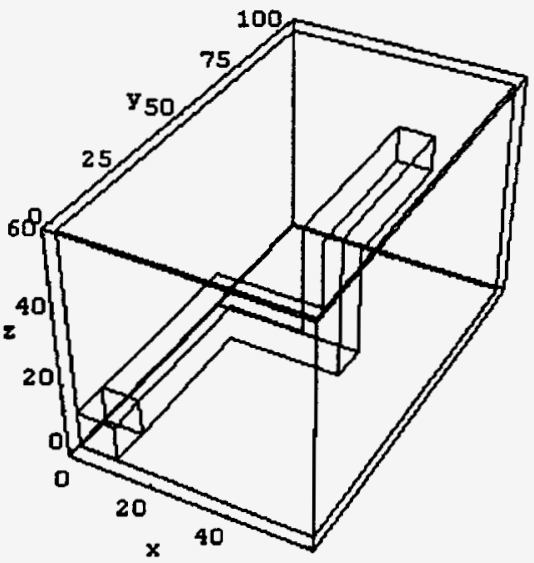

Figure 5 Kobayashi Benchmark Problem 3; dog-leg void duct. $\mathrm{cm}$ between 5 and $55 \mathrm{~cm}$, at $y=95 \mathrm{~cm}$ and $z=35 \mathrm{~cm}$.

The PENTRAN model has four $z$-levels of thickness $10 \mathrm{~cm}$ each, which in turn are partitioned into 5 axial meshes. The $x-y$ plane is partitioned into $6 \times 7$ coarse meshes. These meshes are refined further into a total of 12581 meshes. Note that the mesh refinement is done mainly along the diagonals between the source and positions of comparison.

Figure 6 shows the 3-D flux distribution as projected onto the mesh distribution. Comparison of the PENTRAN and analytical fluxes for the cases $3 \mathrm{~A}$ to $3 \mathrm{C}$ positions indicates: Along the $y$-axis, case $3 \mathrm{~A}$, the two solutions differ by $<$ $6 \%$. Along the $x$-axis, case $3 \mathrm{~B}$, a maximum difference of $<\sim 9 \%$ occurs at $x=55 \mathrm{~cm}$; Along the $x$-axis, case $3 \mathrm{C}$, a maximum difference of $<26 \%$ occurs at $x=15 \mathrm{~cm}$.

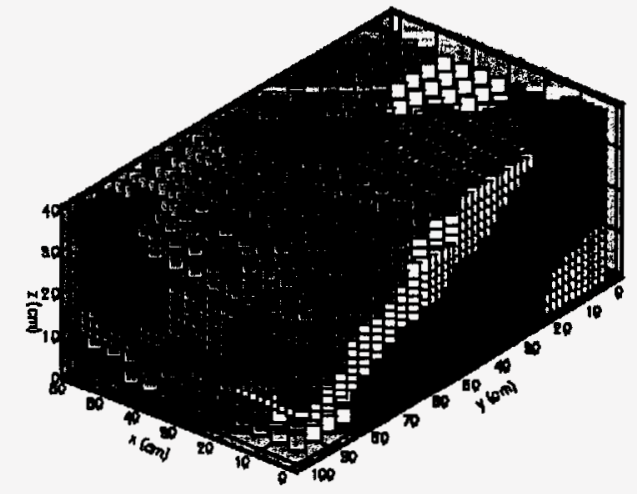

Figure 6 Flux distribution in Problem 3 with pure absorber.

\section{II.3 SUMMARY AND CONCLUSIONS}

This report presented PENTRAN solutions for the Kobayashi simple 3-D benchmark problems. These problems are simple in energy dependency (i.e., one group), and scattering anisotropy (i.e., $\mathrm{P}_{0}$ ), but they are challenging for the discrete ordinates method because fluxes are needed in the void, and a purely absorbing or $50 \%$ scattering shield that can extend several mfps from a localized fixed-source. Considering the limited number of directions of an S20 level-symmetric quadrature set, PENTRAN results are in excellent agreement with the reference analytical or 
Monte Carlo solutions. The largest difference between the PENTRAN and reference solutions occurs at large distances $(7-8 \mathrm{mfps})$ from the source $(<27 \%)$, and at corner discontinuities between the shield and void regions $(<37 \%)$.

The excellent agreement of the PENTRAN results with the reference solutions can be attributed to the $u$ se of u nique $n$ umerical formulations and features a vailable in PENTRAN, a long with selection of an appropriate spatial mesh to allow for the "smearing" of angular fluxes. Note that simple mesh coarsening in order to provide "smearing" does not lead to "good" solutions; appropriate numerical formulations are needed in order to preserve the solution accuracy. For example, we have demonstrated for the selected mesh arrangement and highest level-symmetric quadrature order of $S_{20}$, accurate solutions are achieved only when both adaptive differencing and TPMC formulations are coincidentally applied. In problem 3 , with the pure absorber shield, the solution deviates from analytical values by a factor of 2 when the TPMC formulation is not applied.

Finally, this study indicates that the PENTRAN's numerical formulations can significantly reduce the "ray-effect" with a relatively low quadrature order of S20. 


\section{DEVELOPMENT OF NEW MULTIGRID ALGORITHMS FOR ACCELERATION OF SOLUTION CONVERGENCE, AND TESTED THESE ALGORITHMS FOR DIFFERENT REAL-LIFE PROBLEMS.}

\section{III.1 Introduction}

In problems with optically thick regions and high scattering ratios (c-ratio $=\sigma_{s} / \sigma_{t}$ ), particles that are making a large number of scattering collisions in a single energy group contribute significantly to scalar flux distribution. Consequently, the convergence of the source iteration (SI) method can become very slow. Several techniques such as Rebalance [Miller, 1978], diffusion synthetic acceleration (DSA) [Alcouffe, 1977] and multigrid (MG) [Barnett, 1989] methods have been devised to remedy the slow convergence of the SI method for both shielding and criticality problems.

Rebalance techniques (System Rebalance (SR) and Coarse Mesh Rebalance (CMR)) use the fact that the converged solution must satisfy the neutron conservation (or balance) equation. By imposing this balance condition on the unconverged solution over coarse regions of the problem domain, it is possible to obtain an iteration procedure that may result in faster convergence to the correct solution. Rebalance techniques are effective for deep-penetration problems, however two difficulties are associated with them: i) They suffer from convergence instability; ii) Selection of an optimum coarse-mesh in the rebalance methods is usually difficult, especially in parallel computing environments where spatial domain decomposition is imposed. The Partial Current Rebalance (PCR) [Sjoden and Haghighat, 1996a] method reduces this instability by introducing a damping parameter.

Unlike the rebalance methods, the DSA method works well in eigenvalue problems with high cratios. However it is not as effective in low c-ratio shielding problems. In the DSA method, transport solution is used to correct terms in the diffusion equation, and the diffusion solution is used to obtain an improved source for the transport equation. In this method, a diffusion formulation consistent with the $S_{N}$ formulation is required. This means that if the differencing scheme of the transport equation is changed, a new formulation has to be derived for the diffusion solver. Derivation of consistent diffusion formulations becomes difficult especially in three-dimensional (3-D) geometries and with an adaptive differencing strategy [Sjoden and Haghighat, 1997].

In the remaining of this Chapter, we present: i) a discussion on the general multigrid methods, ii) the new angular multigrid methods implemented in the PENTRANTM code [Briggs, 1987], iii) numerical tests using a 3-D benchmark problem, and v) results and analysis.

\section{III.2. GENERAL MULTIGRID METHODS}

In multigrid methods, a sequence of coarse and fine-grids is used to remove different modes of error from the estimate of the solution. The problem converges when the error remaining in the solution estimate is less than some predefined tolerance. We can express the solution (i.e. angular flux) and the associated error as a function of frequency rather than space or direction by applying the Fourier transform [Nowak et al.,1988]. This representation facilitates the 
understanding of how the components of the error are removed by iterations. Assume a coarse and a fine discretization of the same domain as shown in Figure 7. The error modes behave differently on the two grids. The lowfrequency error on the fine-grid becomes a high-frequency error on the coarse-grid. If we were to solve the transport equation on this coarser grid, then we would have a good approximation to the lowfrequency components of fine-grid solution. If we can couple the coarse- and fine-grid solutions, then the convergence rate on the finegrid will be governed only by the high-frequency errors. Since the high frequency errors are attenuated more rapidly than the lowfrequency errors, we have effectively accelerated the overall convergence.
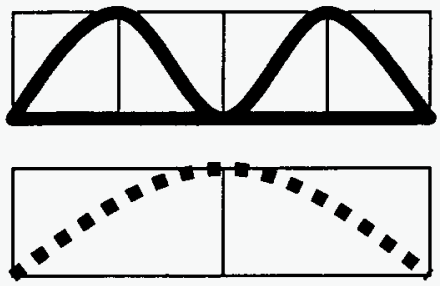

Figure 7 High and low frequency errors on fine- and marse-mrid

Several different types of multigrid methods have been developed [Kucukboyaci and Haghighat, 2000b], [Kucukboyaci and Haghighat, 1999], [Kucukboyaci and Haghighat, 2001a,b] and tested in different disciplines. Few of the examples are $V$-cycle, $W$-cycle, Nested iteration and /-cycle. Multigrid methods have been applied to transport calculations by using it directly for the $S_{N}$ equations [Barnett, 1989], [Sjoden and Haghighat,1996b], and/or for the DSA equations [Alcouffe, 1991]. Many of these applications have been limited to one or two-dimensional problems due to large memory requirements. Recently, an angular multigrid formulation has been developed for highly anisotropic scattering, especially for charged-particle transport problems, both in 1-D and 3-D geometries [Morel and Manteuffel, 1991], [Pautz et al., 1999]. Our method described in this report is different in the sense that it is more general and can be applied to any shielding and criticality calculations.

\section{III.3. ANGULAR MULTIGRID SCHEMES}

\section{Simplified Angular Multigrid (SAM)}

The first angular multigrid scheme we have developed is a /-cycle, which is called the Simplified Angular Multigrid (SAM) scheme. In the SAM scheme, a global approximate solution (i.e., angular fluxes) is obtained on a coarse angular grid (e.g., $\mathrm{S}_{4} / \mathrm{P}_{0}, \mathrm{P}_{1}$ ), and then this solution is projected onto a fine angular grid (e.g., $S_{10} / P_{5}$ ) filtering out the low frequency error components. Effectively, the calculation on the coarse-grid provides preconditioning for the fine-grid iterations.

Using a two-grid approach, PENTRAN first performs a group source iteration/sweep over a coarse angular grid denoted by $\Omega^{2 \mathrm{~h}}$. The zeroth moment balance equation is given by:

$\frac{\left|\mu_{m}\right|^{2 h}}{\Delta x}\left(\Psi_{o u t, x}^{2 h}-\Psi_{i n, x}^{2 h}\right)+\frac{\left|\eta_{m}\right|^{2 h}}{\Delta y}\left(\Psi_{o u t, y}^{2 h}-\Psi_{i n, y}^{2 h}\right)+\frac{\left|\xi_{m}\right|^{2 h}}{\Delta z}\left(\Psi_{o u t, z}^{2 h}-\Psi_{i n, z}^{2 h}\right)+\sigma_{A}^{2 h}=q_{A}^{2 h}$

where $q_{A}^{2 h}$ includes scattering, external and fission sources. Inner iterations on $\Omega^{2 \mathrm{~h}}$ are continued until convergence is achieved (Note that, convergence on the coarse-grid is less strict 
compared to fine-grid $\left(\Omega^{\mathrm{h}}\right)$ ). Then, all the coarse angular fluxes are projected onto the fine angular grid:

$$
\widetilde{\Psi}^{h}=P^{2 h \rightarrow h} \Psi^{2 h}
$$

Here, $P^{2 h \rightarrow h}$ is the projection operator (coarse angular grid to fine angular grid). For this projection, we select the angular flux on a particular direction on $\Omega^{2 \mathrm{~h}}$ that is closest to a direction on $\Omega^{\mathrm{h}}$. The angle between direction vectors of $\Omega^{2 \mathrm{~h}}$ and $\Omega^{\mathrm{h}}$ can be written in terms of direction cosines:

$$
\cos \left(\alpha_{m, n}^{2 h \rightarrow h}\right)=\mu_{m}^{2 h} \mu_{n}^{h}+\eta_{m}^{2 h} \eta_{n}^{h}+\xi_{m}^{2 h} \xi_{n}^{h} \quad \text { where } \quad \begin{aligned}
& n=1, \ldots N(N+2) \in \Omega^{h} \\
& \\
& m=1, . . M(M+2) \in \Omega^{2 h}, M<N
\end{aligned}
$$

By finding the minimum of these angles $\alpha_{m, n}^{2 h \rightarrow h}$, we determine the closest direction.

In case there is more than one minimum angle, the angular fluxes are determined by performing simple arithmetic mean of the fluxes in these directions.

In order to conserve particles, we must guarantee that the integral quantities (i.e., scalar fluxes) rendered on both coarse and fine angular grids are equal. In order to achieve balance we normalize the projected angular fluxes:

$$
\Psi_{n}^{h}=\widetilde{\Psi}_{n}^{h} \frac{\sum_{m=1}^{M(M+2)} w_{m}^{2 h} \Psi_{m}^{2 h}}{\sum_{n=1}^{N(N+2)} w_{n}^{h} \widetilde{\Psi}_{n}^{h}}
$$

Using the projected angular fluxes, the scattering source and the boundary angular fluxes (in case of spatial domain decomposition) on the fine-grid are updated. Then, the iterations/sweeps are continued on the fine angular grid $\left(\Omega^{\mathrm{h}}\right)$ until convergence is achieved.

\section{Nested Iteration (NI)}

A variation of the SAM scheme is the Nested Iteration, in which we use successively refined multiple angular grids (e.g., $\Omega^{8 \mathrm{~h}}, \Omega^{6 \mathrm{~h}}, \Omega^{4 \mathrm{~h}}, \Omega^{2 \mathrm{~h}}, \Omega^{\mathrm{h}}$ ). We start on the coarsest angular grid (e.g. $\Omega^{8 h}$ ) and solve for angular fluxes within certain convergence tolerance. These angular fluxes are then used as the initial solution for the next finer grid. This process is continued until we converge on the finest grid $\left(\Omega^{\mathrm{h}}\right)$. Note that the convergence tolerance for the coarser grids should not be as small as the finer grids. This issue is examined later in this paper.

Both SAM and Nested have the following features:

- Efficiency: The number of operations per mesh is significantly lower for the coarse angular grid compared to the fine angular grid.

- Memory requirement: SAM and Nested Iteration are not cyclic algorithms; all angular flux arrays are overwritten when iterations upgrade to a finer grid. Therefore, no extra memory is required.

\section{V-Cycle}


The V-cycle algorithm uses a two-grid scheme. We, first perform an iteration on the fine angular grid, and compute the difference between the previous and the current iteration scattering sources for each cell and direction. This difference is called the residual. Residuals are then expanded into moments to be used as source on the coarse angular grid. We then perform a sweep on the coarse angular grid to render the error terms. Using the closest direction approach, these error terms are projected back to the fine-grid to update the angular fluxes and the scattering source. We, then proceed to the next iteration with the updated source. We cycle between the two grids until a converged solution on the fine angular grid is obtained. The following algorithm summarizes the angular multigrid V-Cycle:

- Sweep $H^{h} \Psi^{h}=q^{h}$ on $\Omega^{h}$ with the initial guess $\Psi^{h}$

- Compute residual $r^{h}=q^{h}-q_{\text {old }}^{h}$

- Sweep $H^{2 h} e^{2 h}=P^{h \rightarrow 2 h} q^{h}$ on $\Omega^{h}$ with the initial guess $e^{h}=0$

- Update fluxes $\widetilde{\Psi}^{h}=\Psi^{h}+P^{2 h \rightarrow h} e^{2 h}$ and scattering source $q^{h} \rightarrow \widetilde{q}^{h}$

- Sweep $H^{h} \Psi^{h}=\widetilde{q}^{h}$ on $\Omega^{h}$ with the initial guess $\widetilde{\Psi}^{h}$

where $H$ is the transport operator, $r$ is the residual, $e$ is the error, $P$ is the projection operator. $h$ and $2 h$ represent fine- and coarse-grids respectively, and tilde represents the updated values.

Unlike the SAM and Nested Iteration schemes, in the V-cycle scheme, the angular fluxes on the fine-grid are saved, since they are updated with the error terms computed on the coarse-grid. Saving the angular fluxes imposes extra memory requirement, which can be compensated by the possible increase in the rate of solution convergence. It is important to note that all the angular multigrid algorithms described are compatible with the parallel memory structure and the adaptive differencing strategy of the PENTRAN ${ }^{\mathrm{TM}}$ code. Fig. 8 depicts the V-cycle and its combinations with SAM and NI.

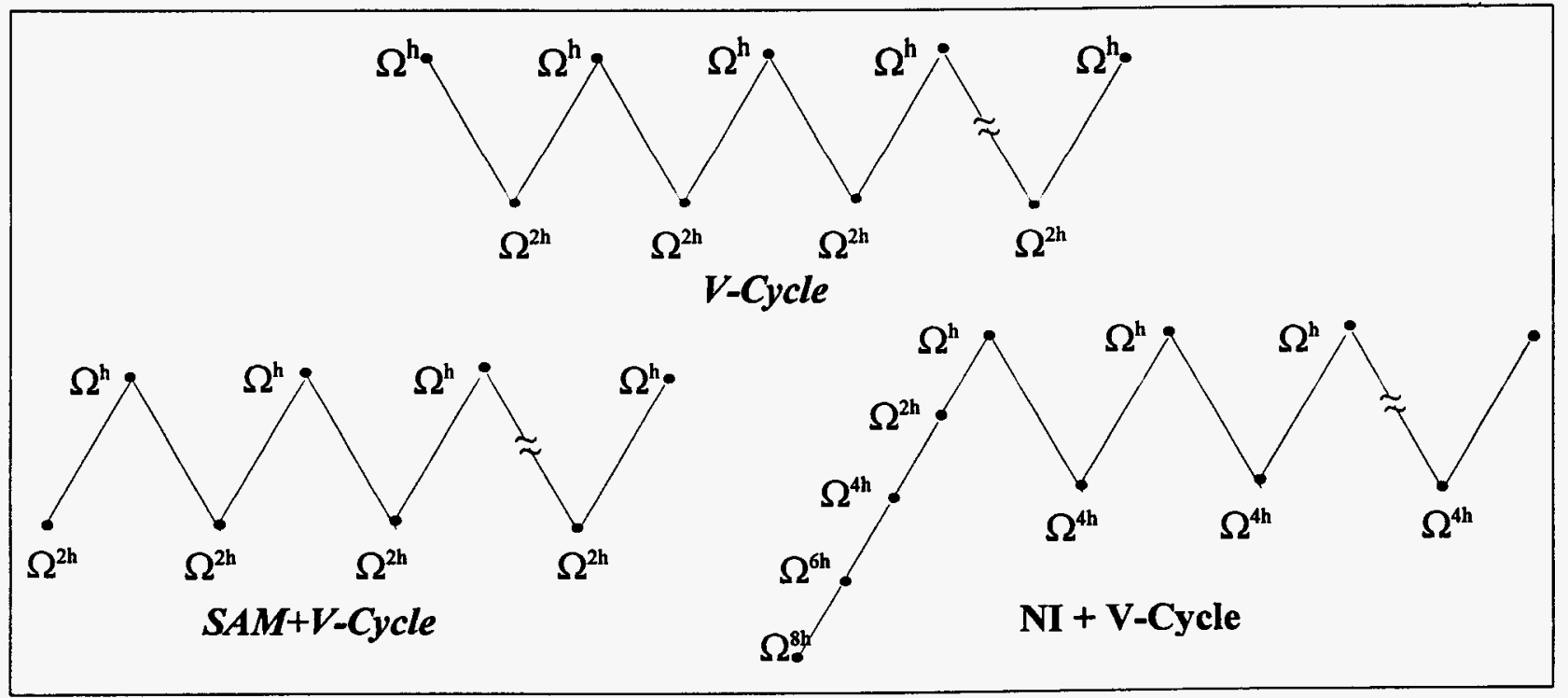

Figure $8 \mathrm{~V}$-cycle and its combination with SAM or NI. 


\section{III.4 NUMERICAL TESTS}

In this section, we measure the performance of the angular multigrid schemes for different problem parameters such as c-ratio, coarse and fine angular grid quadrature orders, and convergence tolerances. We utilize problem 1 of the Kobayashi 3-D deterministic transport benchmark problems [Kobayashi, 1996]. For this study, we have used an $S_{20}$ level-symmetric angular quadrature set. Scattering is isotropic, and we have analyzed cases with different c-ratios ranging from 0.6 to 0.99 . Table 1 shows the total cross sections, fine mesh thickness, and differencing schemes used in different regions of the problem. Detailed studies [Haghigat and Sjoden, 1999] on this benchmark problem have demonstrated that the Directional Theta Weighted (DTW) differencing scheme is adequate for the source and void regions (small flux gradients), while the Exponential Directional Weighted (EDW) differencing scheme is adequate for the absorber regions (large flux gradients).

Table 1 Kobayashi benchmark problem specifications

\begin{tabular}{|c|c|c|c|}
\hline Region & $\sigma\left(\mathrm{cm}^{-1}\right)$ & $\Delta \mathrm{x}-\Delta \mathrm{y}-\Delta \mathrm{z}$ & Differencing Scheme \\
\hline Source & $1 . e-01$ & $1.0 \mathrm{~cm}$ & DTW \\
\hline Void & $1 . e-04$ & $10.0 \mathrm{~cm}$ & DTW \\
\hline Absorber & $1 . e-01$ & $10.0 \mathrm{~cm}$ & EDW \\
\hline
\end{tabular}

For parallel processing of this problem, we have partitioned the angular domain into four subdomains ( 2 octants/ processor) and processed them on 4 processors of the LIONX parallel PC Cluster at Penn State University.

\section{III.5 RESULTS AND ANALYSIS}

Here, we investigate how convergence tolerances, c-ratio, and quadrature order on the coarse and fine-grids affect the performance of the SAM, NI, V-cycle and the combined algorithms. We also compare the effectiveness these new algorithms to that of PCR. We measure the performance by iteration and CPU speed-up. Note that

$$
\text { Iteration Speedup }=\frac{\text { Number of fine grid iterations without acceleration }}{\text { Number of fine grid iterations with acceleration }}
$$

while

$$
C P U \text { Speedup }=\frac{C P U \text { time without acceleration }}{C P U \text { time with acceleration }}
$$

\section{Effect of Coarse- and Fine-grid Tolerances}

In this test, we have determined the effect of coarse- and fine-grid tolerances for the SAM scheme. For a fixed c-ratio of $0.9, S_{20}$ for fine-grid and $S_{10}$ for coarse-grid, we have varied the coarse-grid convergence tolerance in a range of $1 . e-01$ to $1 . e-06$ and the fine-grid tolerance in the range of 1.e-03 to 1.e-06. For all fine-grid tolerances, we observe that SAM scheme becomes 
more effective with a tighter coarse-grid tolerance. However, note that for the coarse-grid tolerances below 1.e-04, no further speed-up is obtained. We also observe that as the tolerance becomes tighter on the fine-grid, SAM becomes less effective, regardless of the coarse-grid tolerance. It is also concluded that in order to obtain the maximum CPU speedup, the coarse-grid tolerances should be in the range of 1.e-03-1.e-04.

\section{Effect of c-ratio}

Using a fixed fine-grid convergence tolerance of 5.e-04, $S_{20}$ for the fine-grid and $S_{10}$ for the coarse-grid, we have performed tests for SAM with different c-ratios ranging from 0.6 to 0.99 . The coarse-grid convergence tolerance is varied from 5.e-01 to 5.e-04. It is demonstrated that SAM becomes more effective with the increasing c-ratio, resulting in a significant acceleration as high as $\sim 7.8$. Further, SAM outperforms PCR by a factor of $\sim 2.6$ in iteration speed-up, however it performs similar to PCR for CPU speed-up.

Our results indicate that using PCR with SAM decreases the number of coarse-grid iterations, thereby increasing the overall efficiency of the angular scheme. This combination accelerates the calculation by a factor of $\sim 4$ while PCR alone achieves a speedup $\sim 2.9$ for c-ratio of 0.99 and the coarse-grid tolerance of 1.e-03. We have repeated this test using the NI scheme combined with PCR. In NI, we have started the calculations on $S_{4}$ grid, progressing to $S_{10}$ and applied PCR on each grid. These analyses yielded similar results as the SAM and PCR combination for large cratios, but the effectiveness decreases for small c-ratios and the small coarse-grid tolerances. Further, our results suggest that the performance of NI is less sensitive to the coarse-grid tolerance.

\section{Effect of Coarse- and Fine-grid Quadrature Orders}

For a fixed c-ratio of 0.9 and $S_{20}$ for the fine-grid, we have performed tests for the SAM scheme using a range of coarse-grid quadrature orders and convergence tolerances. Examining the iteration speed-up behavior, we observe that there is a relation between the iteration speed-up, coarse-grid quadrature order and tolerance. As we either increase the quadrature order or decrease the tolerance, we get better speedups. The behavior of the CPU speed-up is rather different. Beyond $S_{8}$ and tolerances below 5.e-03, the efficiency of SAM decreases due to a higher computational effort on the coarse-grid.

Table 2 provides information on relation between the coarse- and the fine-grid quadrature orders in terms of the CPU speed-up. This test has been performed for a c-ratio of 0.6 , and coarse and fine-grid convergence tolerances of 5.e-02 and 5.e-04, respectively. Table 2 indicates that for an effective acceleration for problems with fine-grid quadrature orders up to $S_{10}$, the coarse-grid quadrature order should be close to fine-grid quadrature order. Beyond $S_{10}$ for the fine-grid, the coarse-grid quadrature orders should not be greater than $S_{8}$ or $S_{10}$.

\section{Combinations of Angular Multigrid Formulations}

In Table 3, we summarize various combinations of the angular multigrid formulations and the PCR acceleration. This test has been performed for a c-ratio of 0.6 , coarse and fine-grid 
Table 2 Relation between coarse- and fine-grid quadrature orders for the SAM scheme

\begin{tabular}{|c|c|c|c|c|c|c|c|c|}
\hline \multirow{2}{*}{$\begin{array}{c}\text { Coarse-grid } \\
\text { Quadrature } \\
\text { Order }\end{array}$} & \multicolumn{8}{|c|}{ Fine-grid Quadrature Order } \\
\hline & $\mathbf{S}_{6}$ & $\mathbf{S}_{\mathbf{8}}$ & $S_{10}$ & $S_{12}$ & $\mathbf{S}_{14}$ & $S_{16}$ & $S_{18}$ & $\mathbf{S}_{\mathbf{2 0}}$ \\
\hline $\mathbf{S}_{4}$ & 1.12 & 1.11 & 1.13 & 1.19 & 1.21 & 1.17 & 1.22 & 1.21 \\
\hline$S_{6}$ & - & 1.22 & 1.16 & 1.29 & 1.24 & 1.21 & 1.27 & 1.24 \\
\hline $\mathbf{S}_{8}$ & - & - & 1.24 & 1.26 & 1.23 & 1.30 & 1.37 & 1.34 \\
\hline$S_{10}$ & - & - & - & 1.29 & 1.29 & 1.27 & 1.32 & 1.40 \\
\hline$S_{12}$ & - & - & - & - & 1.26 & 1.28 & 1.30 & 1.25 \\
\hline$S_{14}$ & - & - & - & - & - & 1.24 & 1.30 & 1.28 \\
\hline$S_{16}$ & - & - & - & - & - & - & 1.25 & 1.21 \\
\hline$S_{18}$ & - & - & - & - & - & - & - & 1.14 \\
\hline
\end{tabular}

${ }^{\mathrm{a}} c$-ratio $=0.9$, coarse- and fine-grid convergence tolerances of 5.e-02 and 5.e-04, respectively.

tolerances and quadrature orders of 5.e-02/5.e-04, and $S_{10} / S_{20}$, respectively. For the Nested Iteration (NI), we have started on $S_{4}$, gradually upgrading to $S_{10}$. Table 3 indicates that angular multigrid formulations combined with PCR become very effective. SAM combined with PCR reduces the CPU by a factor of $\sim 3.43$, while PCR alone reduces by a factor of $\sim 2.38$. The combination of V-cycle, SAM and PCR can significantly reduce number of fine-grid iterations, however, because of the high cost of V-cycle, is not as effective in reducing the CPU time.

Table 3 Comparison of speedups obtained by combined formulations ${ }^{\text {a }}$

\begin{tabular}{|l|c|c|}
\hline & $\begin{array}{c}\text { ITERATION } \\
\text { SPEED-UP }\end{array}$ & $\begin{array}{c}\text { CPU } \\
\text { SPEED-UP }\end{array}$ \\
\hline NO ACCELERATION & 1.00 & 1.00 \\
\hline PCR & 2.43 & 2.38 \\
\hline SAM & 2.76 & 1.52 \\
\hline SAM+PCR & 5.41 & 3.24 \\
\hline NI & 1.83 & 1.37 \\
\hline NI+PCR & 5.62 & 3.39 \\
\hline V-cycle & 1.74 & 1.28 \\
\hline V-cycle+PCR & 4.87 & 3.43 \\
\hline V-cycle+SAM & 3.24 & 1.57 \\
\hline V-cycle+SAM+PCR & 7.30 & 3.39 \\
\hline V-cycle+NI & 2.76 & 1.58 \\
\hline V-cycle+NI+PCR & 6.95 & 2.45 \\
\hline
\end{tabular}

${ }^{a} c$-ratio $=0.6, S_{10}$ for coarse-grid (for SAM and V-cycle), $S_{4}$ to $S_{10}$ for the NI coarse-grids, $S_{20}$ for fine-grid, and coarseand fine-grid convergence tolerances of 5.e-02 and 5.e-04, respectively. 


\section{III.6 USE OF THE NEW MULTIGRID ALGORITHMS FOR A REAL-LIFE BWR PROBLEM}

Kucukboyaci and Haghighat (2000a) have successfully used the new angular multigrid algorithms for a BWR core shroud problem. It is concluded that the angular multigrid algorithms become more effective with increasing values of C-ratio. Using the SAM and NI provide better initial solutions for the V-Cycle algorithm. Moreover, the use of PCR with both SAM/NI with VCycle results in significant reduction in the number of iteration and CPU time. The combination of NI, V-Cycle, and PCR yields the lowest number of iterations and the highest value of speedup. For example, for the BWR problem, the number of fine-grid iterations reduces by a factor of $\sim 7.5$ and the CPU time reduces by a factor of $\sim 4.1$. Further detail on timing and iteration analyses are provided in Kucucboyaci, 2001. 


\section{DEVELOPMENT OF NEW SCHEMES FOR GENERATION OF ANGULAR QUADRATURE SETS FOR BEING ABLE TO SIMULATE HIGHLY ANGULAR DEPENDENT PROBLEMS}

\section{IV.1 Introduction}

A major issue affecting the accuracy of the $S_{N}$ method is selecting an appropriate set of directions. In order to preserve the physics, the general criteria for generating quadrature sets, are preserving symmetry and the moments of the direction cosines.

In the level-symmetric quadrature set $\left(\mathrm{LQ}_{\mathrm{N}}\right)$, the discrete directions are chosen to be fully symmetric with respect to all coordinate axes. There is a total of $\mathrm{N}(\mathrm{N}+2)$ directions on the unit sphere, where $\mathrm{N}$ is the $\mathrm{S}_{\mathrm{N}}$ order. The weight associated to each direction is evaluated by satisfying the moment conditions for direction cosines. The $L Q_{N}$ technique, however, is limited to order 20, because beyond which some of the weights become negative [Carlson and Lathrop, 1965].

In order to prevent this difficulty, B.G. Carlson proposed the Equal Weight Quadrature set $\left(E Q_{N}\right)$, which is characterized by positive weights for any $S_{N}$ order [Carlson, 1971b]. In the $E Q_{N}$ technique, all the direction weights are set equal to $w=1 /[\mathrm{N}(\mathrm{N}+2)]$.

Other quadrature sets have been derived, by relaxing the constraints imposed by the $\mathrm{LQN}_{\mathrm{N}}$ method. For this purpose, the Gauss quadrature technique has been used to derive quadrature sets based on the Legendre and Chebyshev polynomials, which yield positive weights. In a recent study, two new quadrature sets $\left(\mathrm{UE}_{\mathrm{N}}\right.$ and $\left.U \mathrm{UG}_{N}\right)$ have been derived. The $U E_{N}$ quadrature set is derived by uniformly partitioning the unit sphere in the number of direction defined by the $S_{N}$ order [Carew and Zamonsky, 1999]. The $\mathrm{UG}_{\mathrm{N}}$ quadrature set selects the ordinates along the $\mathrm{z}^{-}$ axis as roots of Legendre polynomials.

In this study, we investigate other techniques which allow generation of quadrature sets of orders higher than 20 [Longoni and Haghighat, 2001b].

\section{IV.2 THE LEGENDRE EQUAL-WEIGHT $\left(\mathbf{P}_{\mathrm{N}}-\mathrm{EW}\right)$ QUADRATURE SET}

In order to develop a quadrature set which is not limited to order $S_{20}$, we have investigated the Gauss-Legendre quadrature technique. In this quadrature set we utilize the same arrangement of directions as the $L Q_{N}$, but the directions and weights are evaluated differently. Given the $S_{N}$ order for the discrete set of directions, we obtain the Legendre polynomials applying the following recursive formulation:

$$
\begin{aligned}
(j+1) P_{j+1}=(2 j+1) \xi P_{j}-j P_{j-1} \quad \text { for } \mathrm{j}=0 . . \mathrm{N} \\
-1<\xi<1 \quad P_{-1}(\xi)=0 \quad P_{0}(\xi)=1
\end{aligned}
$$

The ordinates, i.e. $\xi$, along the $z$-axis are the roots of the Legendre polynomials given by Eq. 5 . Once we have evaluated the ordinates along $\mathrm{z}$-axis we obtain the weights associated to each level with the following recursive formula: 


$$
w_{i}=\frac{2}{\left(1-\xi_{i}^{2}\right)\left[\left(\frac{d P_{N}}{d \xi}\right)_{\xi_{i}}\right]^{2}}, \quad i=1 \ldots \frac{N}{2}
$$

And the weight associated with each direction is given by:

$$
p_{i, j}=\frac{w_{i}}{j} \quad i=1 \ldots \frac{N}{2}
$$

where $j=1, \frac{N}{2}+1-i$ is the number of directions with equal weights on the $i^{t h}$ level.

In order to evaluate the azimuthal angle on each level, we equally divide a 90 degrees angle by the number of angular intervals (i.e., N/2-i+2) between directions. In Fig. 9, we show the directions selected by $P_{N}-E W$ technique for a $S_{16}$ quadrature set.

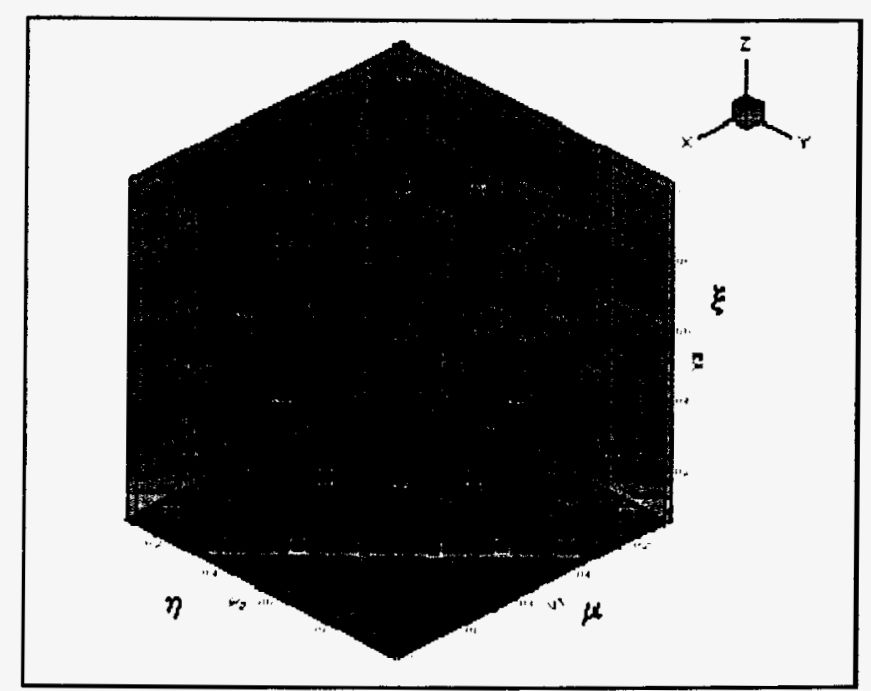

Figure 9 Discrete directions selected on one octant with $\mathrm{S}_{16} \mathrm{P}_{\mathrm{N}}$-EW quadrature set

\section{IV.3 THE LEGENDRE-CHEBYSHEV $\left(\mathbf{P}_{\mathrm{N}}-\mathrm{T}_{\mathrm{N}}\right)$ QUADRATURE SET}

In the $\mathrm{P}_{\mathrm{N}}-\mathrm{T}_{\mathrm{N}}$ methodology, similar to $\mathrm{P}_{\mathrm{N}}-\mathrm{EW}$, we set the $\xi$ levels on the $z$-axis equal to the roots of Legendre polynomials, but for the azimuthal angles on each level we use the roots of the Chebyshev $T_{N}$ polynomials of first kind. The Chebyshev polynomials of first kind have the following formulation:

$$
T_{l}[\cos (\omega)] \equiv \cos (l \omega)
$$

The Chebyshev polynomials are orthogonal and satisfy the following condition:

$$
\begin{aligned}
& \int_{-1} d y T_{l}(y) T_{k}(y)\left(1-y^{2}\right)^{-1 / 2}=\left\{\begin{array}{l}
0, l \neq k \\
\pi, l=k=0 \\
\pi / 2, l=k \neq 0
\end{array}\right. \\
& y=\cos (\omega)
\end{aligned}
$$


Again, using the ordering of the $\mathrm{LQ}_{\mathrm{N}}$ quadrature set, we set the azimuthal angles on each level using the following formulation:

$$
\omega_{l, i}=\left(\frac{2 l-2 i+1}{2 l}\right) \frac{\pi}{2} \quad \omega_{l, i} \in\left(0, \frac{\pi}{2}\right)_{, i=1 . . . l}
$$

In Eq. $10, l$ is the level number. The level and point weights are generated in the same way as for the $\mathrm{P}_{\mathrm{N}}-\mathrm{EW}$. Both $\mathrm{P}_{\mathrm{N}}-E W$ and $\mathrm{P}_{\mathrm{N}}-\mathrm{T}_{\mathrm{N}}$ sets do not present negative weights for $\mathrm{S}_{\mathrm{N}}$ orders higher than 20. Fig. 10 shows an example for a $P_{N}-T_{N}$ quadrature set of order 30 .

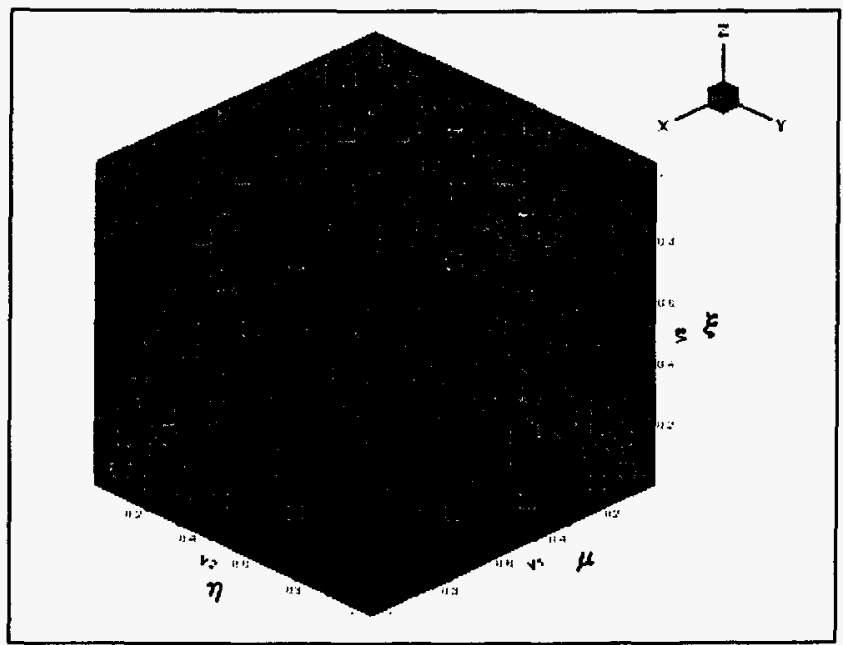

Figure 10 Discrete directions selected on one octant with $S_{30} P_{N}-T_{N}$ quadrature set

\section{IV.4 THE REGIONAL ANGULAR REFINEMENT (RAR) TECHNIQUE}

The RAR technique has been developed for those problems where the angular flux and/or source are highly peaked. The RAR technique differs from OS, rather than splitting an ordinate, a subdomain inside a $\mathrm{P}_{\mathrm{N}}-\mathrm{T}_{\mathrm{N}}$ quadrature set is partitioned into a large number of solid angles [Longoni and Haghighat, 2002a, b, c]. Inside the subdomain we fit an additional $P_{N}-T_{N}$ quadrature set. One of the main advantages of the RAR technique is the conservation of the moments of the direction cosines, with consequent increased accuracy. The $P_{N}-T_{N}$ is derived by setting the $\xi$ levels, on the $\mathrm{z}$-axis of the unit sphere, equal to the roots of Legendre polynomials $\left(\mathrm{P}_{\mathrm{N}}\right)$. The azimuthal angles on each level are set equal to the roots of the Chebyshev polynomials $\left(\mathrm{T}_{\mathrm{N}}\right)$ of first kind. The Chebyshev polynomials of first kind have the following formulation:

$$
T_{l}[\cos (\omega)] \equiv \cos (l \omega)
$$

The Chebyshev polynomials are orthogonal and satisfy the following condition:

$$
\begin{aligned}
& \int_{1}^{l} d y T_{l}(y) T_{k}(y)\left(1-y^{2}\right)^{-1 / 2}=\left\{\begin{array}{l}
0, l \neq k \\
\pi, l=k=0 \\
\pi / 2, l=k \neq 0
\end{array}\right. \\
& y=\cos (\omega)
\end{aligned}
$$

By using the ordering of the $L Q_{N}$ quadrature set, we set the azimuthal angles on each level using the following formulation: 


$$
\omega_{l, i}=\left(\frac{2 l-2 i+1}{2 l}\right) \frac{\pi}{2} \quad \omega_{l, i} \in\left(0, \frac{\pi}{2}\right), i=1 \ldots l
$$

In Eq. 13, $l$ is the level number. The $P_{N}-T_{N}$ set does not present negative weights for $S_{N}$ orders higher than 20. In Fig. 11 we show a $\mathrm{S}_{12} \mathrm{P}_{\mathrm{N}}-\mathrm{T}_{\mathrm{N}}$ quadrature set biased with $\mathrm{RAR}$ [Longoni and Haghighat, 2002b].

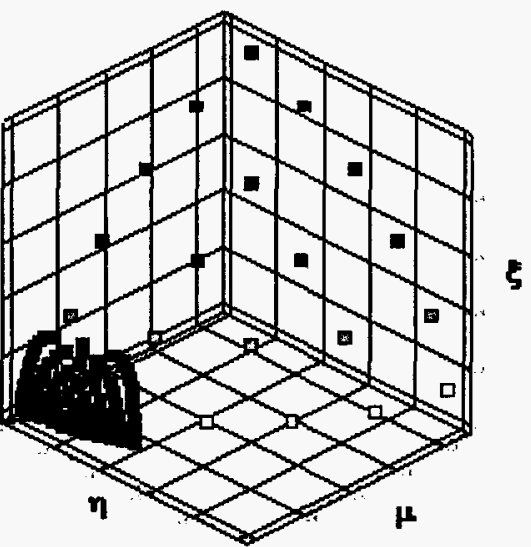

Figure $11 \mathrm{~S}_{12} \mathrm{P}_{\mathrm{N}}-\mathrm{T}_{\mathrm{N}}$ biased with $\mathrm{RAR}$

\section{IV.5 APPLICATION OF THE NEW QUADRATURE SETS}

We implemented the new quadrature sets into the PENTRAN code, and simulated a simplified CT-Scan model. The model is composed of a highly directional source ("fan" beam), a large region of air and a detector, as shown in Fig. 12.

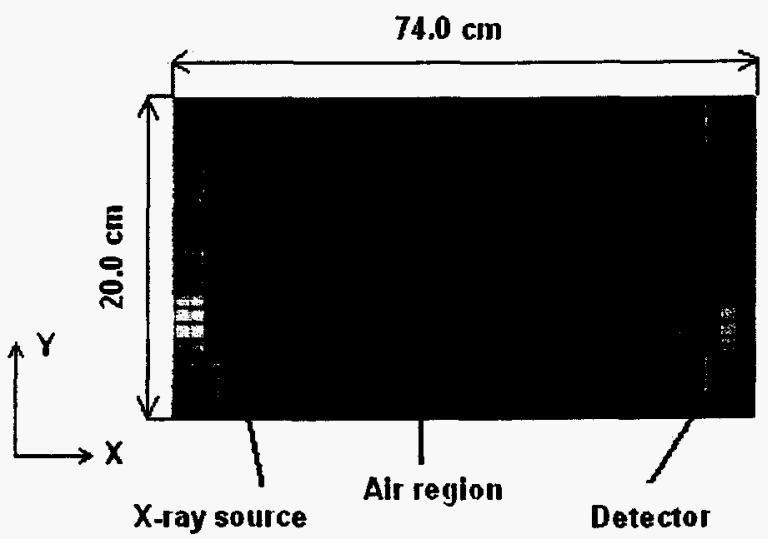

Figure 12 Simplified CT-Scan model.

Because of the presence of large void regions and a directional source, the solution of the transport equation is affected by ray-effects. One remedy is to use higher order quadrature sets with biasing such as ordinates splitting (OS) [Longoni and Haghighat, 2001a] and RAR technique [Longoni and Haghighat, 2002a]. For the purpose of this manuscript, we compare the results to a reference solution obtained with a $S_{80} P_{N}-T_{N}$ quadrature set. The RAR technique has been applied to a $S_{12} P_{N}-T_{N}$ quadrature set; the biased region on the octant extends from $z=0.0$ to $z=0.3$ and the azimuthal angle spans from 0 to 25 degrees. 
In the biased region, we used a $S_{26} P_{N}-T_{N}$ quadrature set. The $S_{12} P_{N}-T_{N}$ biased with $S_{26} P_{N}-T_{N}$ resulted in 110 directions per octant. The $S_{80} P_{N}-T_{N}$ quadrature set yielded 820 directions per octant. The $S_{20} L Q_{N}$ quadrature set yielded 55 directions per octant. We have chosen these parameters based on the knowledge of the X-rays fan-beam. In Fig. 13, we show the scalar flux at $x=30.0 \mathrm{~cm}$ from the source, along the $y$ axis.

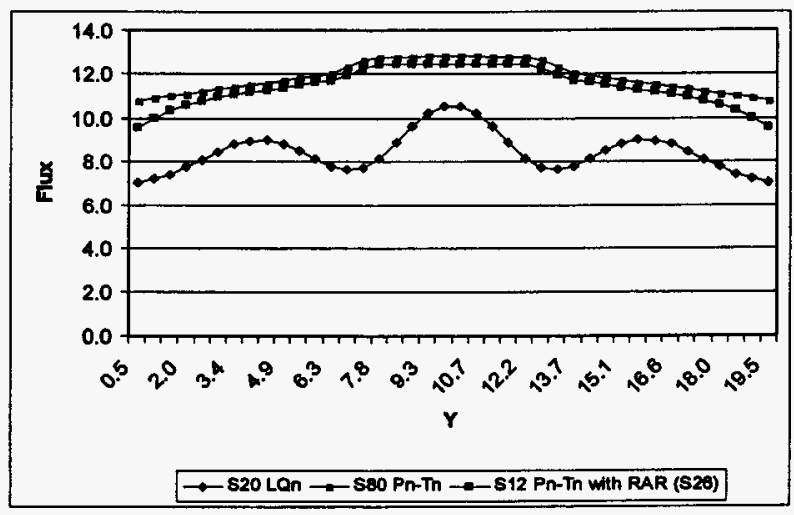

Figure 13 Scalar flux at $x=30.0 \mathrm{~cm}$ from the source.

It is clear that RAR technique is in agreement with the $S_{80} P_{N}-T_{N}$ quadrature set, while the solution obtained with the $S_{20} L Q_{N}$ quadrature set is inaccurate and affected by ray-effects. In Fig. 14 we show the scalar flux at $x=40.0 \mathrm{~cm}$ along the $y$-axis. At these positions, the RAR technique still yields accurate results compared to the $S_{80} P_{N}-T_{N}$ solution.

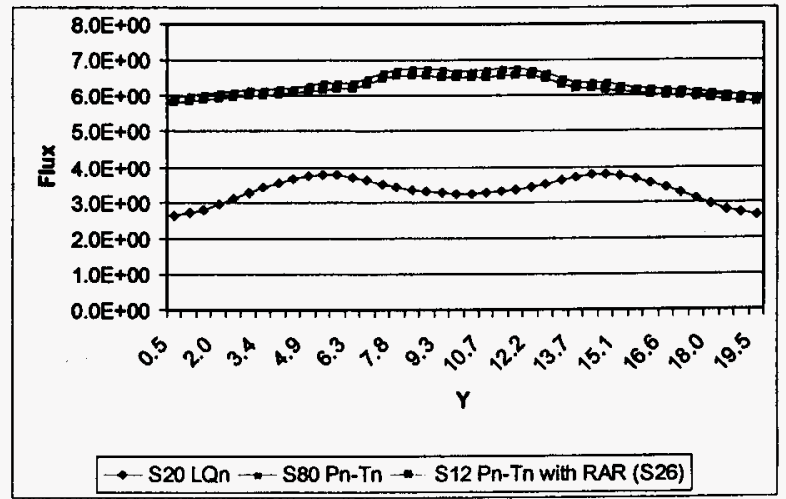

Figure 14 Scalar flux at $\mathrm{x}=40.0 \mathrm{~cm}$ from the source.

In Fig. 15, we show the scalar flux at the detector position along the $y$-axis. As shown in Fig.15, the $S_{12}$ $P_{N}-T_{N}$ biased with $S_{26}$ shows excellent agreement with $S_{80} P_{N}-T_{N}$. Fig. 15 also demonstrates that the $S_{20}$ $\mathrm{LQ}_{\mathrm{N}}$ quadrature set underpredicts the flux distribution at the detector position by more than one order of magnitude. It is worth noting that the range of oscillation occurring in the predicted flux distributions from $S_{80}$ and $S_{12}$ with RAR is less than $10 \%$. 


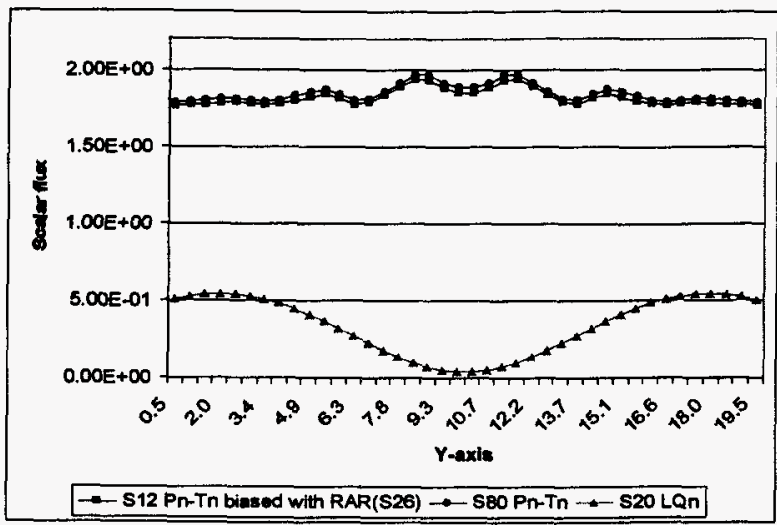

Figure 15 Scalar flux at detector position.

In Table 4, we show a comparison of the computational time involved in the simulation.

Table 4 Computational time and memory required for the simulation ${ }^{1}$.

\begin{tabular}{|c|c|c|}
\hline Quadrature Set & Directions $^{2}$ & CPU Time(sec) \\
\hline $\mathrm{S}_{80} \mathrm{P}_{\mathrm{N}}-\mathrm{T}_{\mathrm{N}}$ & 6560 & 682.9 \\
\hline $\mathrm{S}_{12} \mathrm{P}_{\mathrm{N}}-\mathrm{T}_{\mathrm{N}} \mathrm{RAR}\left(\mathrm{S}_{26}\right)$ & 880 & 56.3 \\
\hline $\mathrm{S}_{20} \mathrm{LQ}_{\mathrm{N}}$ & 440 & 25.0 \\
\hline
\end{tabular}

We can observe that the RAR technique greatly reduces the computational time, by more than one order of magnitude compared to $S_{80}$, while resulting in accurate solution.

Fig. 16 shows a 3-D plot of the scalar flux obtained with the $L Q_{N}$ quadrature set; the ray-effects increase as we move far away from the source.

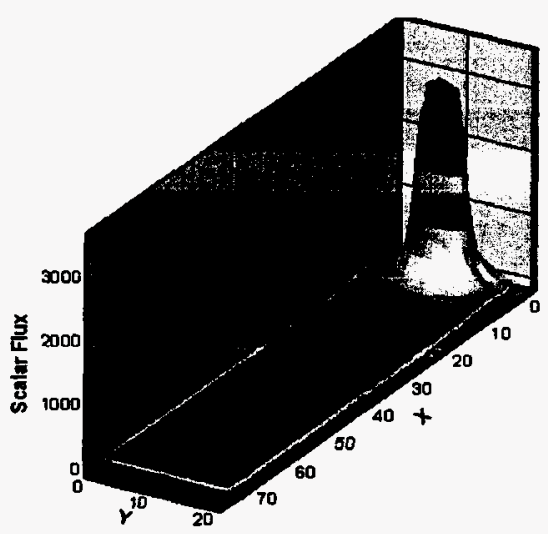

Figure 16 Diagram of the scalar flux calculated with $S_{20} L Q_{N}$ quadrature set.

\footnotetext{
${ }^{1}$ These results have been achieved on a PC-Workstation with $1 \mathrm{GHz}$ Pentium III processor and 256 MBytes RAM.

${ }^{2}$ Total number of directions on the unit sphere.
} 
Fig. 17 shows the scalar flux calculated with the $S_{12}$ with $S_{26} R A R$. In these case the solution exhibits small ray-effects, even far away from the source.

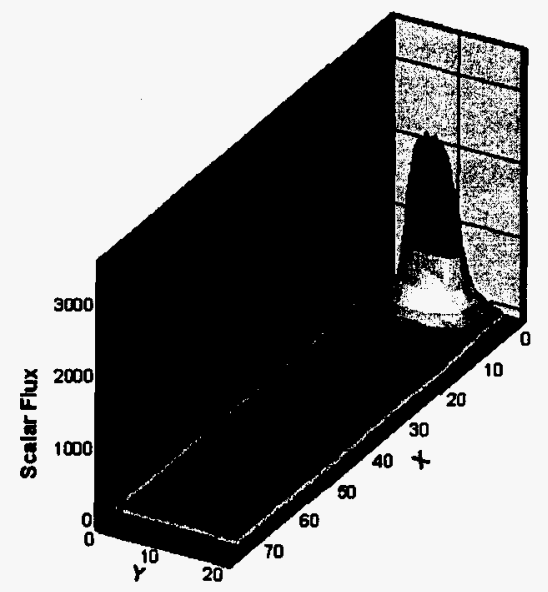

Figure 17 Plot of the scalar flux calculated with $S_{12} P_{N}-T_{N}$ quadrature set biased with $S_{26} R A R$.

\section{IV.5 SUMMARY AND CONCLUSIONS}

We have developed new techniques for generation of angular quadrature sets and biasing for local refinement. We have concluded that for problems with highly localized angular dependencies, the $P_{N}-T_{N}$ algorithm with RAR yields the most accurate solutions in a rather short time. 


\section{DEVELOPED A GENERAL 3-D PARALLEL SIMPLIFIED $P_{L}\left(S_{2} P_{L}\right)$ ALGORITHM FOR ACCELERATION OF THE SN METHODOLOGY.}

\section{V.1 Introduction}

The $\mathrm{SP}_{\mathrm{L}}$ equations were initially proposed by Gelbard [Gelbard et al, 1959] in the early 1960s. Following their introduction, they did not receive much attention due to weak theoretical support. Recently, the $\mathrm{SP}_{\mathrm{L}}$ equations have received more attention because they have been shown to provide more accurate solutions compared to the diffusion equation. Moreover, the theoretical foundations of the $\mathrm{SP}_{\mathrm{L}}$ equations have been significantly strengthened in recent years using a variational analysis approach in the derivation [Lewis and Palmiotti, 1997].

In a previous paper, we derived the 3-D SP $\mathrm{S}_{3}$ equations, starting from the 1-D $\mathrm{P}_{3}$ equations and by applying the Gelbard procedure; we implemented these equations in the $\mathrm{PENSP}_{3}$ (Parallel Environment Neutral-particle $\mathrm{SP}_{3}$ code) [Longoni and Haghighat, 2002d]. The structure of these equations is characterized by a second order elliptic operator, which makes them amenable to a solution with standard iterative techniques, such as preconditioned conjugate gradient methods. In this paper, we will derive the $S P_{L}$ equations, starting from the even-parity form of the 1-D $S_{N}$ $(\mathrm{N}=\mathrm{L}+1)$ transport equations. Morel, Larsen and $\mathrm{McGhee}$ obtained the $\mathrm{SP}_{\mathrm{L}}$ equations in a similar fashion [Morel et al, 1996]. The derivation of the $S P_{L}$ equations starting from the even-parity form of the $S_{N}$ equations presents many advantages that are shared by the alternative derivation we presented in our previous work [Longoni and Haghighat, 2003a]. However, we note a distinctive property in the new derivation; the fluxes are mathematically decoupled on the boundary. This property allowed us to easily modify the $\mathrm{PENSP}_{3}$ code to accommodate an arbitrary $\mathrm{SP}_{\mathrm{L}}$ order.

\section{V.2 NUMERICAL TESTING OF THE SP FORMULATION}

We have selected the NEACRP-L-330 problem 1 from a collection of 3-D benchmark problems [Takeda and Ikeda, 1988], proposed by T. Takeda and H. Ikeda. The purpose of these problems is to validate the accuracy of present transport codes for criticality calculations. Problem 1 consists of a 3-D model of the Kyoto University Critical Assembly (KUCA). The core is composed by 93 w/o enriched U-Al alloy and natural uranium metal plates; the average U-235 enrichment is $9.6 \mathrm{w} / \mathrm{o}$ and the moderator is polyethylene. The core is symmetric about the origin and the dimensions are, in one octant, $15 \mathrm{~cm} \times 15 \mathrm{~cm} \times 15 \mathrm{~cm}$. A control rod (CR) is introduced outside of the core in the reflector region. The model cross sections over $x-y$ plane and $x-z$ plane are shown in Figs. 18 and 19 respectively (dimensions are in centimeters).

The characteristics of this problem make it challenging for the $\mathrm{SP}_{L}$ equations; the presence of the void $r$ egion involves $l$ ong $s$ treaming $p$ aths, $w$ hile the control rod p roduces s teep thermal flux gradients at the interface. We compare the results obtained using the $\mathrm{SP}_{\mathrm{L}}$ and $\mathrm{S}_{\mathrm{N}}$ methods with those presented in the benchmark using the Monte Carlo method. 

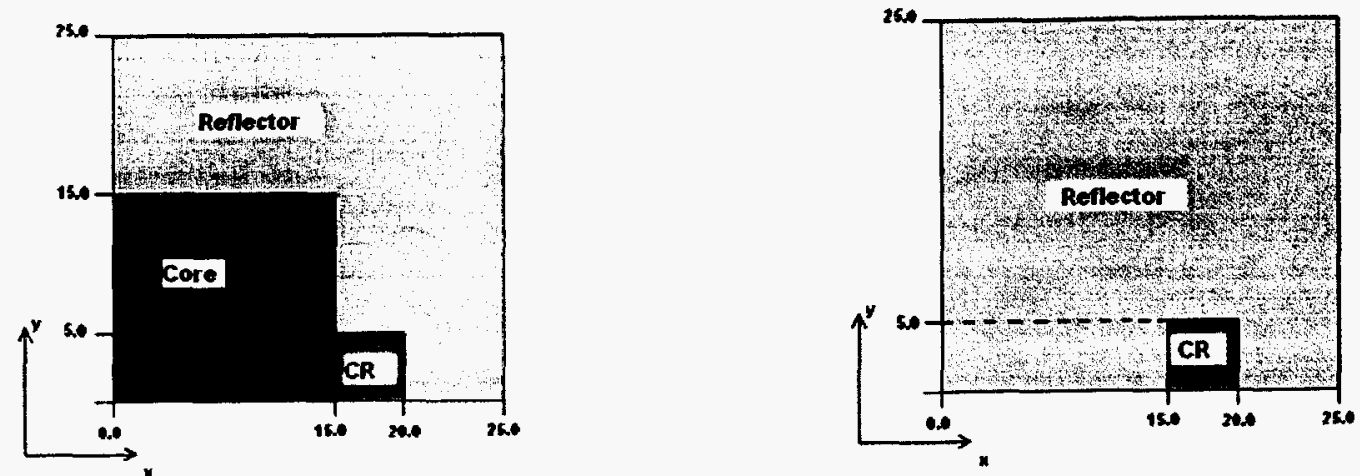

Figure 18 Model cross section on the $x-y$ plane $(l e f t-z=0.0 \ldots 15.0 \mathrm{~cm}$; right $z=15.0 . .25 .0 \mathrm{~cm}$ )

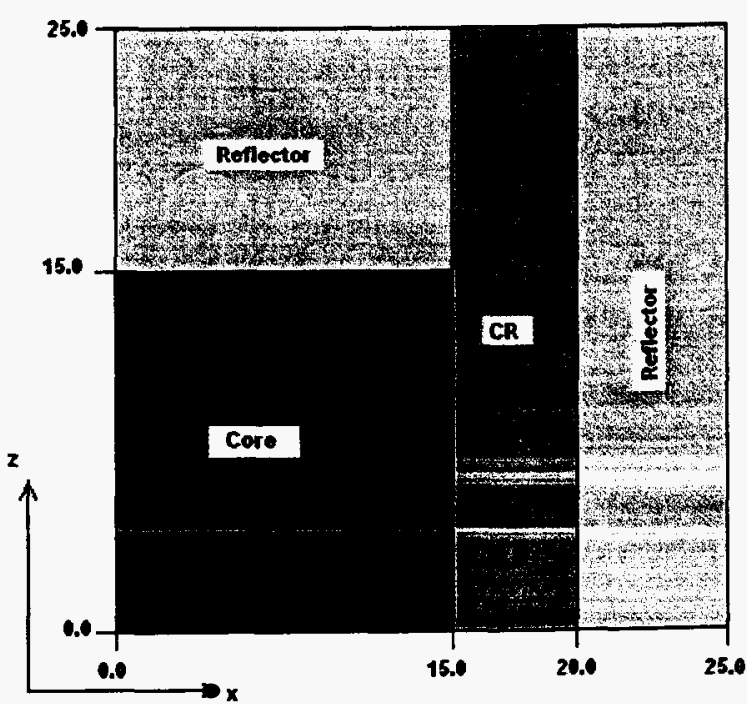

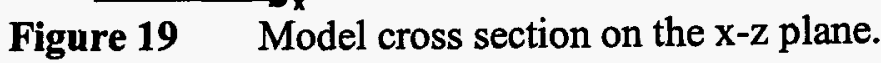

Two cases have been considered, with different configurations of the control rod; in the first case, the control rod is fully withdrawn and region is left empty (voided); in the second case, the control rod is fully inserted into the system. Hence, we evaluated the control rod worth, defined by Eq. 14 .

$$
C R W=\left(\frac{1}{k_{e f f}}\right)_{\text {Case } 2}-\left(\frac{1}{k_{e f f}}\right)_{\text {Case } 1}
$$

We have used the two-group cross sections provided in the benchmark problem; note that the $\mathrm{P}_{1}$ scattering effect has been included by using the transport cross section in place of the total cross section. Table 5 gives the convergence criteria for the inner and outer iterations for the $S_{N}$ and $\mathrm{SP}_{\mathrm{L}}$ calculations. 
Table 5. Criticality eigenvalues

\begin{tabular}{|l|l|l|}
\hline Methodology & Inner Tolerance & Outer Tolerance \\
\hline $\mathbf{S}_{\mathbf{N}}$ & $1.0 \mathrm{E}-4$ & $1.0 \mathrm{E}-5$ \\
\hline $\mathbf{S P}_{\mathbf{L}}$ & $1.0 \mathrm{E}-3$ & $1.0 \mathrm{E}-5$ \\
\hline
\end{tabular}

The model is divided into two coarse $z$-levels; the first $z$-level spans from $z=0.0 \mathrm{~cm}$ to $z=15.0$ $\mathrm{cm}$, while the second $\mathrm{z}$-level ranges from $\mathrm{z}=15.0 \mathrm{~cm}$ to $z=25.0 \mathrm{~cm}$. We used the linear Diamond differencing with zero fixup (DZ) in PENTRAN [Sjoden and Haghighat, 1997], and a linear averaged formulation in $\mathrm{PENSP}_{\mathrm{L}}$. The model is consistently discretized with a $1-\mathrm{cm}$ uniform mesh along the three axes for each method.

In Table 6, we show the criticality eigenvalues and the errors relative to the Monte Carlo solution for both cases, when the control rod is inserted and withdrawn from the reactor.

Table 6. Criticality eigenvalues

\begin{tabular}{|l|l|l|l|l|}
\hline Methodology & $\begin{array}{l}\text { k-effective } \\
\text { (Rod OUT) }\end{array}$ & $\begin{array}{l}\text { Error } \\
\text { (Rod OUT) }\end{array}$ & $\begin{array}{l}\text { k-effective } \\
\text { (Rod IN) }\end{array}$ & $\begin{array}{l}\text { Error } \\
\text { (Rod IN) }\end{array}$ \\
\hline $\mathbf{P}_{\mathbf{1}}$ & 0.92663 & -5.25 & 0.93352 & -3.0 \\
\hline $\mathbf{S P}_{\mathbf{3}}$ & 0.95568 & -2.28 & 0.96281 & 0.04 \\
\hline $\mathbf{S P}_{\mathbf{5}}$ & 0.95639 & -2.21 & 0.96357 & 0.12 \\
\hline $\mathbf{S}_{\mathbf{8}}$ & 0.97705 & -0.097 & 0.96226 & -0.01 \\
\hline Monte Carlo & $0.9780 \pm 0.0006$ & Reference & $0.9624 \pm 0.0006$ & Reference \\
\hline
\end{tabular}

In Table 7, we show the calculated control rod worth.

Table 7. Control Rod Worth

\begin{tabular}{|c|c|}
\hline Methodology & Control Rod Worth \\
\hline $\mathbf{P}_{\mathbf{1}}$ & $-7.96 \mathrm{e}-3$ \\
\hline $\mathbf{S P}_{\mathbf{3}}$ & $-7.75 \mathrm{e}-3$ \\
\hline $\mathbf{S P}_{\mathbf{5}}$ & $-7.79 \mathrm{e}-3$ \\
\hline $\mathbf{S}_{\mathbf{8}}$ & $1.57 \mathrm{e}-2$ \\
\hline Monte Carlo & $1.66 \mathrm{e}-2$ \\
\hline
\end{tabular}

The c ontrol rod w orth c alculated with the $S P_{L}$ methodology is n egative, as reported b y o ther authors [Morel and et al.,1996] and $\mathrm{P}$. Kotiluoto. The $\mathrm{SP}_{\mathrm{L}}$ calculations yield accurate results for the Control Rod-In case, but when the control rod is replaced with the void region, the keffective is underestimated by $\sim 2.2 \%$ with $\mathrm{SP}_{5}$. These results contradict the physics of the problem, because a higher $\mathrm{k}$ eigenvalue is expected when the control rod is withdrawn. The $\mathrm{S}_{8}$ transport calculation provides good accuracy compared to Monte Carlo, with an error of $-0.01 \%$. These results are consistent with the $S_{\mathrm{N}}$ results presented in the Takeda Benchmark. We observe that the $\mathrm{SP}_{L}$ methodology yields more accurate criticality eigenvalues in the Control Rod-In case, compared to the Control Rod-Out case. Hence, we conclude that the $\mathrm{SP}_{\mathrm{L}}$ methodology is more

\footnotetext{
${ }^{3}$ Percentage relative error compared to Monte Carlo.
} 
accurate in representing the transport physics of highly absorbing materials as compared to void regions with long streaming mean free paths.

In Fig. 20, we show the scalar flux (normalized) at $\mathrm{z}=8.0 \mathrm{~cm}$ and $\mathrm{y}=2.5 \mathrm{~cm}$ in group 1 for the Control Rod-Out case (with the void region in place of the control rod). We observe a steeper gradient of the flux obtained with $\mathrm{P}_{1}$, $\mathrm{SP}_{3}$, and $\mathrm{SP}_{5}$ in the proximity of the void region, compared to the $\mathrm{S}_{8}$ solution. This can be attributed to the inability of the $\mathrm{SP}_{\mathrm{L}}$ leakage operator to represent the streaming of particles in void regions. For this problem, $\mathrm{SP}_{\mathrm{L}}$ overestimates the particle leakage in the void, which consequently leads to the underestimation of k-effective, and therefore yields a negative control rod worth.

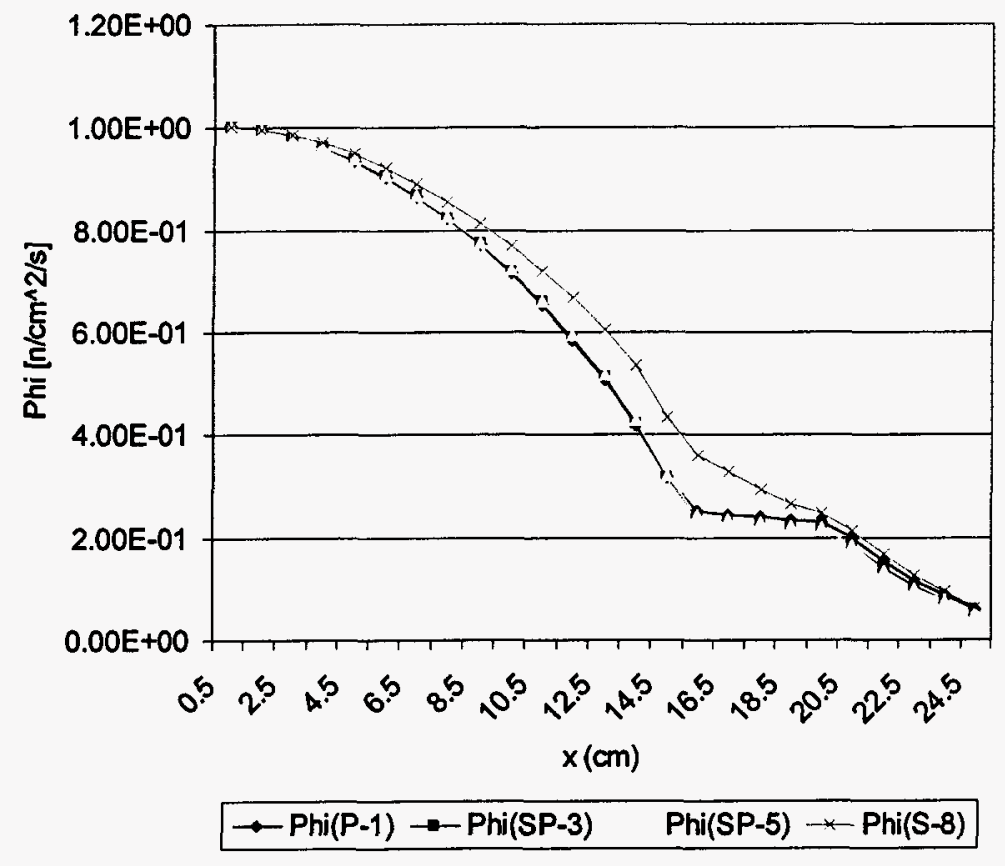

Figure 20 Control Rod OUT - Normalized Flux at $z=8.0 \mathrm{~cm} y=2.5 \mathrm{~cm}$ (Group 1)

The maximum error, obtained with $P_{1}$ method and compared to the $S_{8}$ solution is $\sim 10 \%$ at $x=15$ $\mathrm{cm}$, inside the control rod. In this case, the $\mathrm{SP}_{\mathrm{L}}$ equations cannot resolve the angular dependency introduced by the void region when the control rod is withdrawn, even with $\mathrm{SP}_{5}$ treatment.

In Fig. 21, we show the flux distributions obtained with $\mathrm{P}_{1}, \mathrm{SP}_{3}, \mathrm{SP}_{5}$ and $\mathrm{S}_{8}$ at $\mathrm{y}=2.5 \mathrm{~cm}$ and $\mathrm{z}=8.0 \mathrm{~cm}$, for the case with the Control Rod-In. The $\mathrm{SP}_{\mathrm{L}}$ methodology yields accurate flux distributions compared to the $\mathrm{S}_{\mathbf{8}}$ solution. For the Control Rod-In case, the $\mathrm{P}_{1}, \mathrm{SP}_{3}$ and $\mathrm{SP}_{5}$ equations yield maximum relative errors equal to $15.3 \%, 4.5 \%$ and $3.4 \%$ respectively.

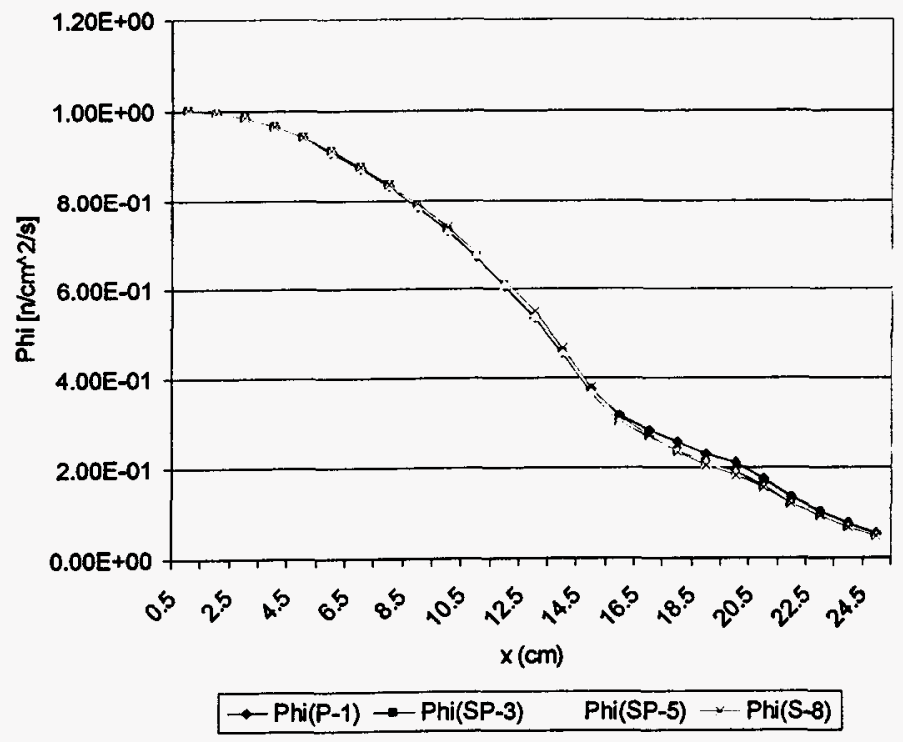

Figure 21 Control Rod IN Normalized Flux at $\mathrm{z}=8.0 \mathrm{~cm}$ $y=2.5 \mathrm{~cm}$ (Group 1) 


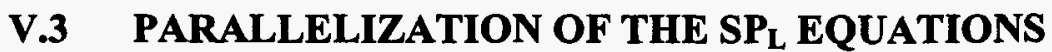

The PENSP $_{\mathrm{L}}$ code has been implemented in a distributed memory architecture using the MPI parallel libraries, based on the moment decomposition strategy [Longoni and Haghighat, 2003a, b]. The $\mathrm{PENSP}_{\mathrm{L}}$ code allocates automatically the arrays containing the matrices for the evenparity angular fluxes on the available processors. During the decomposition phase, we try to preserve load balancing in order to achieve maximum efficiency.

The solution for each moment is computed, and at the end of each iteration, the necessary information is exchanged among the processors. The parallel computer utilized is the PCPEN cluster. The s ystem has 8 processors, with 2 GByte R AM e ach. The head node is an A thlon Pentium IV $1.6 \mathrm{GHz}$ and the remaining 7 nodes have Pentium III $1 \mathrm{GHz}$ processors.

In Tables 8 and 9, we present the timing and parallel performance of the code for the aforementioned 3-D benchmark problem for different $P_{L}$ orders of 3 and 5, without and with use of the Incomplete Cholesky Preconditioning, respectively.

Table 8 - Performance of the PENSP $_{L}$ code for the 3-D benchmark problem (without Incomplete Cholesky Preconditioning is used)

\begin{tabular}{|c|c|c|c|c|c|}
\hline Method & \# processors & $\begin{array}{c}\text { Calc. Time } \\
(\mathbf{s})\end{array}$ & Total time (s) & Speedup & $\begin{array}{c}\text { Efficiency } \\
(\%)\end{array}$ \\
\hline $\mathrm{SP}_{3}$ & 1 & 72 & 73 & - & - \\
& 2 & 56 & 57 & 1.29 & 64 \\
\hline $\mathrm{SP}_{5}$ & 1 & 120 & 121 & - & - \\
& 2 & 69 & 70 & 1.74 & 58 \\
\hline
\end{tabular}

Table 9 - Performance of the PENSP $_{L}$ code for the 3-D benchmark problem (without Incomplete Cholesky Preconditioning is used)

\begin{tabular}{|c|c|c|c|c|c|}
\hline Method & \# processors & $\begin{array}{c}\text { Calc. Time } \\
(\mathbf{s})\end{array}$ & Total time (s) & Speedup & $\begin{array}{c}\text { Efficiency } \\
(\%)\end{array}$ \\
\hline SP $_{3}$ & 1 & 57 & 76 & - & - \\
& 2 & 46 & 57 & 1.33 & 66 \\
\hline $\mathrm{SP}_{5}$ & 1 & 96 & 123 & - & - \\
& 2 & 57 & 68 & 1.80 & 60 \\
\hline
\end{tabular}

The relatively low efficiency obtained is due to the load imbalance caused by the Conjugate Gradient (CG) algorithm which requires different numbers of iterations for different moments. Also, it is important to note that the Incomplete Cholesky preconditioner tends to reduce the differences in the number of iterations needed for the CG algorithm, thereby improving the load balance and parallel efficiency. 


\section{DEVELOPMENT OF THE SP ${ }_{\mathrm{L}}$ SYNTHETIC ACCELERATION METHOD}

\section{VI.1 Introduction}

It is a well known fact that the Source Iteration strategy used to solve the $\mathrm{Sn}$ transport equations is very inefficient for highly reactive materials, where the scattering ratio, defined in Eq. 15, is close to unity [Gelbard and Hageman, 1969].

$$
c=\frac{\sigma_{s}}{\sigma_{t}}
$$

For problems in which the scattering process is dominant, the error reduction per iteration is very small. The Diffusion Synthetic Acceleration (DSA) method [Alcouffe, 1977] has been one of most widely techniques. DSA is effective in problems where angular flux is not highly angular dependent, however, where this condition is not met, the method may converge slowly or diverge [Reed, 1971]. In the current project, we have investigated the possibility of using a higher order approximation such as the $\mathrm{SP}_{L}$ method which can represent the angular flux more accurately.

We have performed a Fourier analysis of the $\mathrm{SP}_{\mathrm{L}}$ synthetic acceleration in a 1-D infinite homogeneous system with $c=0.99$ and calculated the expected spectral radius. In Fig. 22, shows the Fourier analysis of the synthetic acceleration applied to the $S_{N}$ equations.

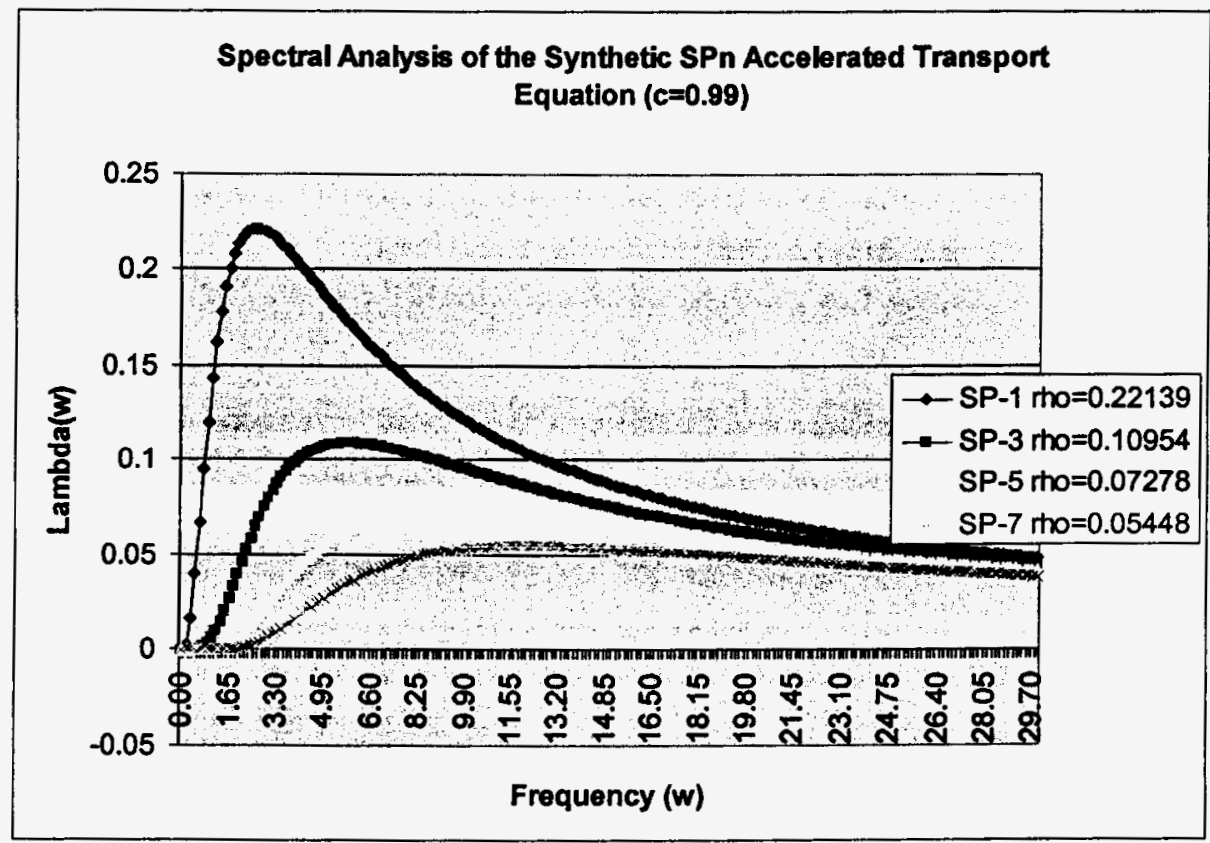

Figure 22 Fourier analysis of the $\mathrm{SP}_{\mathrm{L}}$ synthetic acceleration

The Fourier analysis suggests that a higher order $\mathrm{SP}_{\mathrm{L}}$ operator may reduce the spectral radius and consequently improve the efficiency of the acceleration. 
Using the $\mathrm{PENSP}_{\mathrm{L}}$ code (discussed in Chapter V), we have developed an $\mathrm{SP}_{\mathrm{L}}$ synthetic acceleration algorithm which has been tested within the PENTRAN 3-D discrete ordinates parallel code.

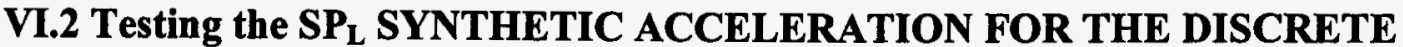 ORDINATE METHOD - PRELIMINARY RESULTS.}

The performance of the $\mathrm{SP}_{\mathrm{L}}$ synthetic acceleration scheme has been tested using the PENTRAN code system for two problems where transport effects are significant.

The first problem considered is a simple box problem (test problem 1), which consists of a fixed source region surrounded by a highly absorbing material. In Fig. 23 we show the problem configuration. The overall dimensions of the system are $20 \times 20 \times 10 \mathrm{~cm}^{3}$.

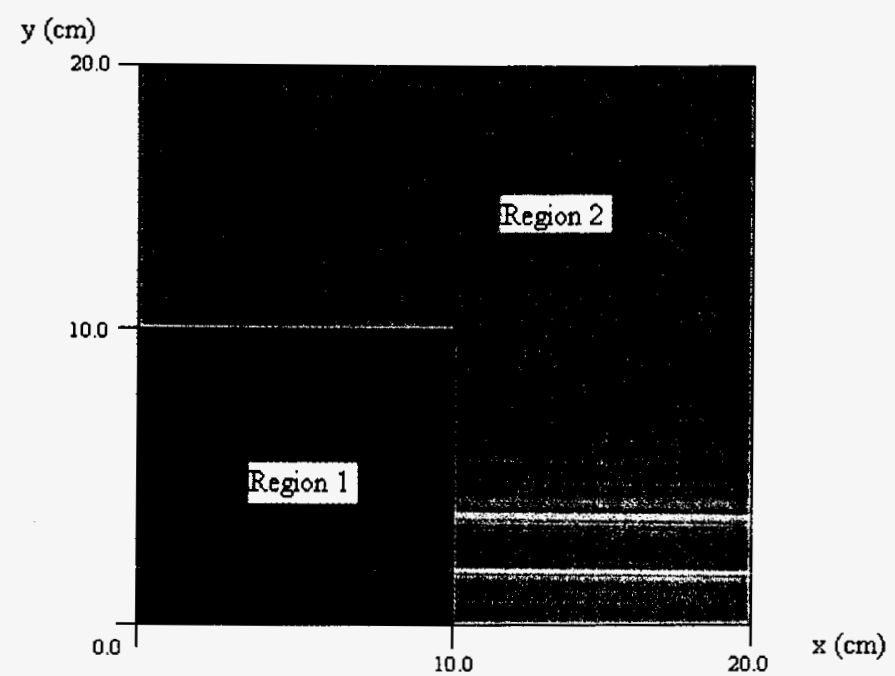

Figure 23 Geometrical configuration of test problem 1.

The total cross section is equal to $1.0\left[\mathrm{~cm}^{-1}\right]$ in both regions, while the scattering cross sections for regions 1 and 2 are $0.9 \mathrm{~cm}^{-1}$ and $0.1 \mathrm{~cm}^{-1}$, respectively. The model is discretized using a constant mesh size of $0.77 \mathrm{~cm}$ along $\mathrm{x}, \mathrm{y}$, and $\mathrm{z}$ axes. The flux convergence tolerance for the $\mathrm{Sn}$ calculation is set to $1.0 \mathrm{e}-5$.

Table 10 gives the performance of the acceleration scheme $\mathrm{SP}_{1}$ (i.e., DSA) and $\mathrm{SP}_{3}$ for an $\mathrm{S}_{8}$ calculation.

Table 10 - Performance of the $\mathrm{SP}_{\mathrm{L}}$ synthetic acceleration for the test problem 1

\begin{tabular}{|c|c|c|}
\hline Method & $\begin{array}{c}\text { Number of Inner } \\
\text { Iterations }\end{array}$ & $\begin{array}{c}\text { Computational Time } \\
(\mathrm{sec})\end{array}$ \\
\hline $\mathrm{S}_{8}$ (unacc.) & 79 & 126.7 \\
\hline $\mathrm{DSA}_{\mathrm{S}}$ & FAILED & $\mathrm{n} / \mathrm{a}$ \\
\hline $\mathrm{SP}_{3} / \mathrm{S}_{8}$ & 21 & 65.3 \\
\hline
\end{tabular}


The above results demonstrate that the DSA method does not converge, while the $\mathrm{SP}_{3}$ acceleration was able to reduce the number of inner iterations by almost a factor of four. The reason for this behavior is the fact that region 2 contains a highly absorbing material for which diffusion theory cannot represent the existing transport effects. Note that the computational time is not reduced proportionally, because of the time spent for $\mathrm{SP}_{3}$ calculations. For example, for this problem, the computation time by is reduced by $\sim 50 \%$.

Fig. 24 shows the relative error in the scalar flux between adjacent iterations. It is apparent that the $\mathrm{SP}_{3}$ synthetic acceleration can significantly reduce the error after a few iterations.

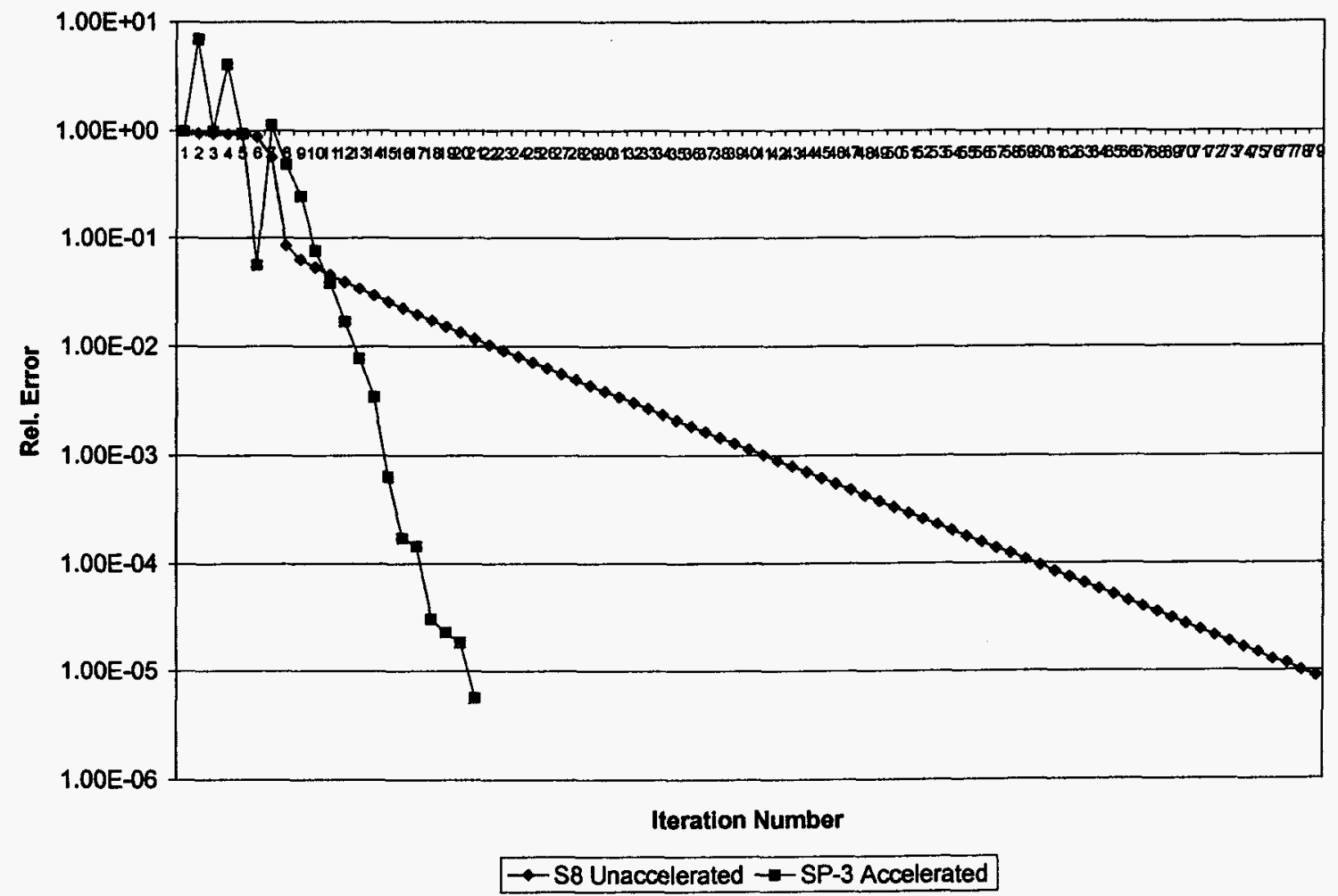

Figure 24 Relative error on the scalar flux between different iterations

The second problem (test problem 2) simulates a small reactor with a control rod inserted in the center. This problem is characterized by strong transport effects and by strong variations of the total cross section between different regions.

Fig. 25 presents the problem configuration along with the mesh and material distributions. The green region (the most inner region) represents the control rod, the red region ( $2^{\text {nd }}$ region) is the fixed source, and the blue region (the most outer region) is the moderator. The overall dimensions of this model are $18 \times 18 \times 10 \mathrm{~cm}^{3}$. The model is discretized into $30,30,10$ fine meshes along $x, y$ and $z$, respectively. The convergence criterion is set to $5.0 \mathrm{e}-5$. 


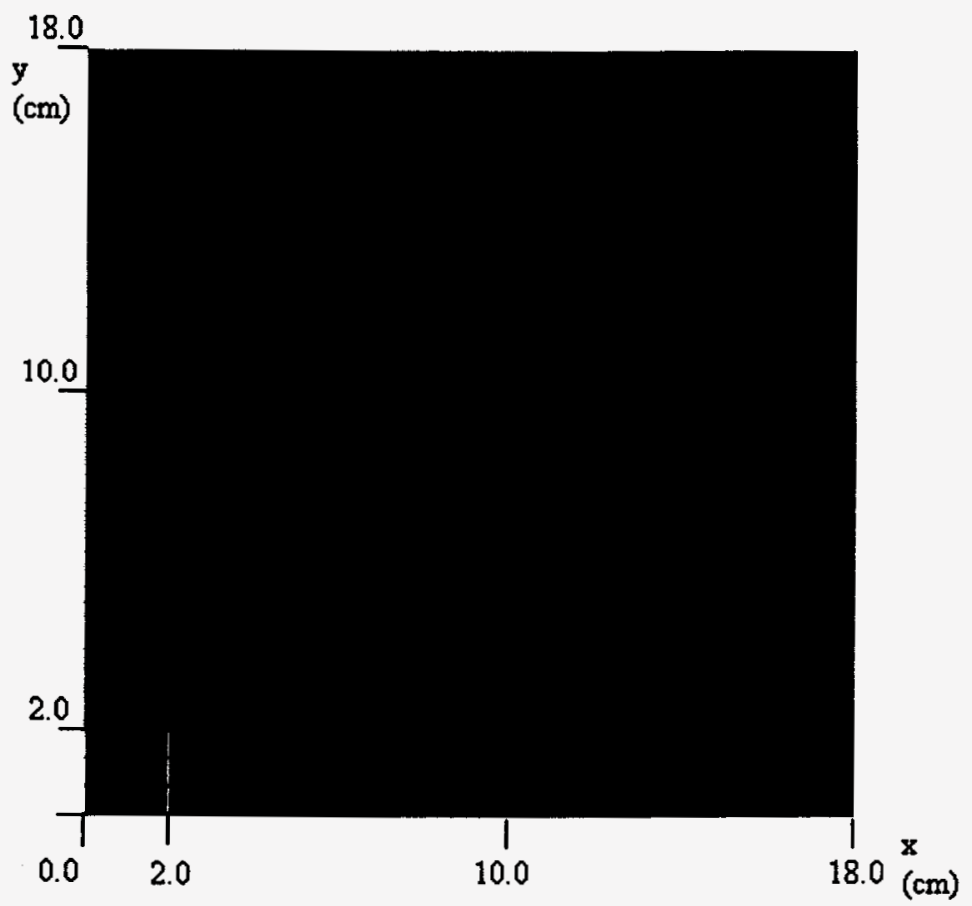

Figure 25 Mesh and material distribution of test problem 2.

Table 11 gives the performance of the $\mathrm{SP}_{\mathrm{L}}$ acceleration method for the $\mathrm{P}_{\mathrm{L}}$ orders of 1 and 3 .

Table 11 - Performance of the $\mathrm{SP}_{\mathrm{L}}$ synthetic acceleration for the test problem 2

\begin{tabular}{|c|c|c|}
\hline Method & $\begin{array}{c}\text { Number of Inner } \\
\text { Iterations }\end{array}$ & Computational Time (sec) \\
\hline $\begin{array}{c}\mathrm{S}_{10} \\
\text { (unaccelerated) }\end{array}$ & 165 & 432.6 \\
\hline $\mathrm{DSA} / \mathrm{S}_{10}$ & FAILED & $\mathrm{n} / \mathrm{a}$ \\
\hline $\mathrm{SP}_{3} / \mathrm{S}_{10}$ & 34 & 169 \\
\hline
\end{tabular}

Again, the $\mathrm{SP}_{1}$ (or DSA) does not converge, while SP3 reduces the number of iterations by a factor $\sim 5$, and consequently the computation time reduces by a factor of $\sim 2.6$

In Fig. 26, we present the behavior of the relative error for the scalar flux between adjacent inner iterations. 


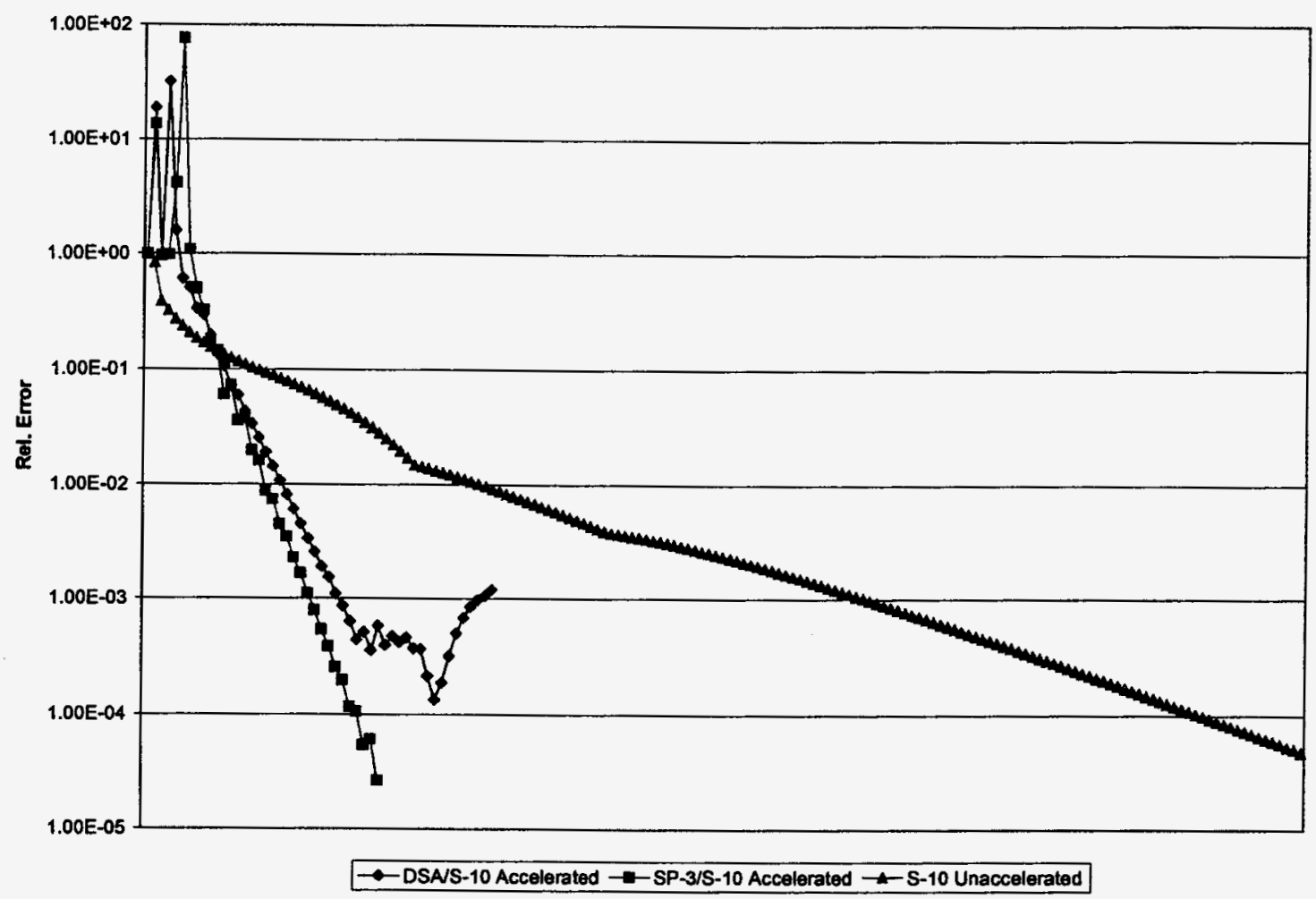

Figure 26 Relative error on the scalar flux between different iterations for the test problem 2.

As expected the $\mathrm{SP}_{3}$ reduces the error significantly in a few iterations, while the DSA starts oscillating. Again, the reason for these oscillations of DSA is the presence of highly heterogeneous medium in which the diffusion approximation cannot simulate the transport effects which are effectively captured with the $\mathrm{SP}_{3}$ formulation. Three-dimensional and 2-D flux distributions shown in Figs. 27 and 28, respectively, indicate presence of steep gradients which cannot be effectively simulated with the diffusion approximation.

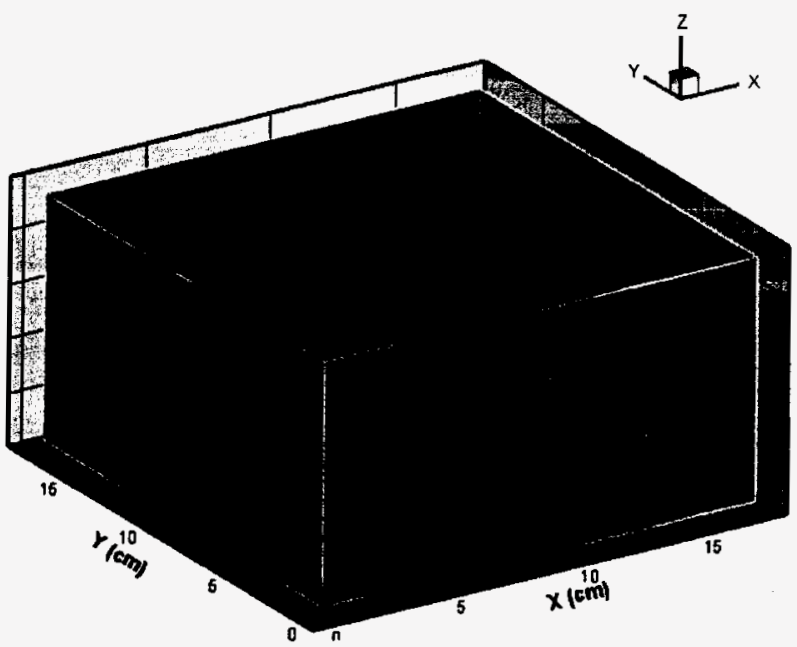

Figure 27 3-D Scalar flux distribution

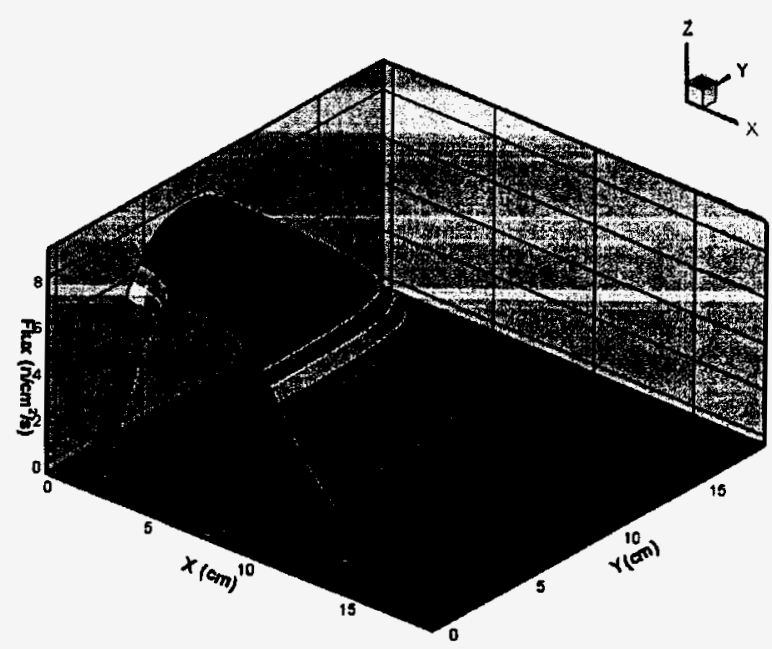

Figure 28
2-D Scalar flux distribution at $\mathrm{z}=5.0 \mathrm{~cm}$ 


\section{DEVELOPMENT OF AN EXPERT SYSTEM FOR MESH GENERATION AND FOR SELECTION OF AN APPROPRIATE DOMAIN DECOMPOSITION ALGORITHM IN A PARALLEL ENVIRONMENT.}

\section{VII.1 Introduction}

The discrete ordinates $\left(\mathrm{S}_{\mathrm{N}}\right)$ method discretizes all of the independent variables including angle, energy and space. One of the challenges for applying this method is generation of an effective spatial mesh distribution. This mesh distribution must not only represent the problem geometry but also account for the problem physics and numerics. For real-life large and complex problems, parallel processing becomes necessary to obtain accurate solutions in a reasonable amount of time [Patchimpattapong and Haghighat, 2000]. Depending on how the independent variables are decomposed, different domain-decomposition strategies (DDSs) may require different amounts of computing resources and results in different parallel performance. To address the above issues, we have developed an expert system for generating an effective spatial mesh distribution for an $S_{N}$ calculation in a parallel environment [Patchimpattapong and Haghighat, 2002a], [Patchimpattapong and Haghighat, 2003a-d]. This expert system comprises two major parts: 1) an algorithm for generating an effective mesh distribution, and 2) an algorithm for selecting an effective parallel DDS.

\section{VII.2 ALGORITHM FOR GENERATION OF AN EFFECTIVE MESH DISTRIBUTION}

The algorithm for generating an effective mesh generation (Patchimpattapong and Haghighat, 2002b] consists of four steps: 1) creation of a geometric model and coarse meshes, 2) estimation of an approximate flux shape, 3) selection of differencing schemes, and 4) generation of a fine mesh distribution. We have developed and tested the methodologies for this 4-step algorithm as discussed below:

- Creation of a geometric model and coarse meshes

We partition a 3-D physical model into $x-y-z$ coarse meshes. For each $z$-level, we utilize AutoCAD to create a 2-D geometric model and coarse mesh layout via the use of layers. Using the VENUS-3 benchmark and the BWR problems, we have demonstrated that our model creation algorithm is a practical tool to facilitate a process of modeling a real-life large and complex system.

- Estimation of an approximate flux shape

We use an uncollided flux distribution as an approximate flux shape for selection of an appropriate spatial differencing scheme. For this, we have developed a parallel code PENFC (Parallel Environment Neutral-Particle First Collision). PENFC is capable of calculating uncollided and first collision fluxes in a 3-D Cartesian geometry in a parallel environment. We have benchmarked PENFC for an uncollided flux calculation against GRTUNCL3D using the dog leg problem, and against PENTRAN [Sjoden and Haghighat, 1997] using the simplified VENUS-3 model. For both, we observe a good agreement in uncollided flux 
shape, while PENFC requires a relatively short computation time. Further, as expected, PENFC demonstrates a high parallel efficiency of $96 \%$ for the simplified VENUS- 3 model.

- Selection of differencing schemes

We fit a function to the uncollided flux distribution obtained from PENFC and apply the method of least squares to determine which differencing scheme best fits the flux distribution. We utilized the VENUS-3 problem to examine the capability of our differencing scheme selection algorithm. For this test problem, the algorithm has correctly predicted differencing schemes for $84 \%$ of the coarse meshes. This demonstrates that an uncollided flux distribution can be used as an approximate flux distribution to determine an appropriate differencing scheme.

- Generation of a fine mesh distribution

To generate a fine mesh distribution, we have developed a serial code PENXMSH. It utilizes the geometric model and coarse mesh layout from Step 1. PENXMSH determines mesh size based on material mean-free-path and assigns a material to a fine mesh by checking its center position against layers of materials in the geometric model. An iterative procedure is employed to examine different mesh sizes in order to achieve the user-specified accuracy in preserving material volumes (masses). We demonstrated, using the VENUS-3 problem, that our fine mesh generation algorithm can yield an effective mesh distribution that requires significantly lesser computation resources while achieving accurate solutions. PENXMSH automatically performs mesh refinement in the vicinity of material discontinuities, which are indicated by a fine-mesh ratio between two adjacent coarse meshes. The current version of the PENXMSH code automatically generates an input file for the pre-processing code PENMSH [Haghighat, 2000] of the PENTRAN code system.

Fig. 28 presents a detailed flowchart for the above four step procedure which leads to an effective mesh distribution. In the flowchart, we have included all the necessary input parameters for each step of the algorithm. 
Figure 28 Flow-Chart for Generation of an effective mesh distribution

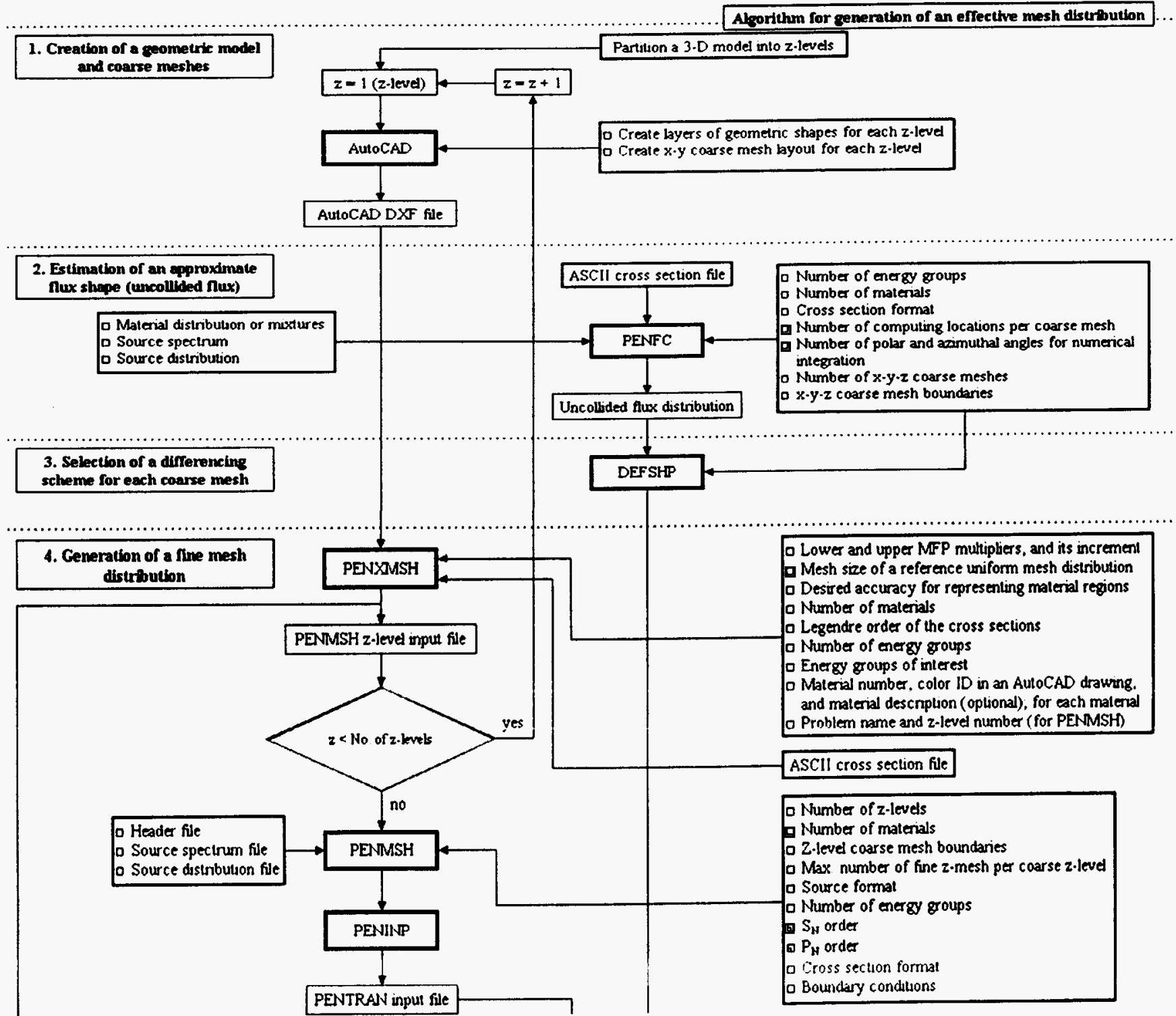

List of abbreviations

Box color definditons

D DXF - Drawnig Interchange fus

- User.spectified infortation

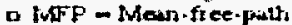

- DDS - Damien decompostion strategy

- DVEC - Decormposiluon veetors

- DTW - Direeloond Thera-Waghsed durferencung scheme

- EDW - Esponential Drectional Wagnted deferenctag scheme

- CPCM - Computation-ta-communication tume ratio

o DCP - Degze-of-coupling among processors

D PPI - Parall-peformance.index 
For parallel processing, we have identified four factors that affect the parallel performance. These are: 1) number of processors and memory available per processor, 2) load balance, 3) granularity, and 4) degree-of-coupling (DCP). We have developed methodologies for estimating these factors within the PENTRAN code system as described below:

- Estimation of the memory required per processor

In PENTRAN, the majority of memory is used for storing angular fluxes and flux moments. These arrays are allocated based on the number of local coarse meshes, local energy groups, and local sweep octants on each processor. As a result, different DDSs may require different amounts of memory. We utilize a mapping algorithm available in PENTRAN to estimate the memory requirement.

- Estimation of the load balance

Different numbers of fine meshes per coarse mesh and/or different differencing schemes causes load imbalance. Fine mesh distribution and differencing schemes can be obtained from the mesh generation algorithm.

\section{- Estimation of the granularity}

We measure granularity in terms of computation-to-communication time ratio (CPCM) in a transport sweep. To simulate a transport sweep without performing the transport calculation, we need the following information: fine mesh distribution, differencing schemes, computation time of each differencing scheme, computation time of scattering source, and communication time of a transport sweep. Fine mesh distribution and differencing schemes can be obtained from the mesh generation algorithm. To estimate the computation time of a differencing scheme, we perform a serial calculation using a simple model with the differencing scheme of interest. We utilize the scattering source calculation algorithm available in PENTRAN to estimate the computation time of scattering source. We estimate the computation times of the differencing schemes and scattering source only once for each computing platform. We have created a communication structure as it is used in PENTRAN to measure the time spent for message passing in a parallel environment. Load imbalance (caused by the different numbers of fine meshes per coarse mesh and different differencing schemes) introduces a waiting time, which effectively increases the communication time of the transport sweep. By using the aforementioned information, we can estimate the computation and communication times of the transport sweep and hence the CPCM.

\section{- Estimation of the DCP}

The DCP is defined as the maximum contribution to the total source in a sub-domain from other processors. To calculate DCP, we obtain an angular flux distribution, partial currents, and a source distribution from a serial calculation of a small model. This small model is created by coarsening meshes and reducing quadrature order. We weight the maximum DCP of each group by its corresponding scattering- or c-ratio, and sum them to obtain the overall DCP of the problem. 
The CPCM gives the relation of computation and communication, while the DCP reflects the convergence (numerical) behavior of the parallel algorithm. To obtain an index that accounts for both factors, we define a parallel-performance-index (PPI) as a ratio of the CPCM to the DCP. Fig. 29 shows a flow-chart for selection of an appropriate domain decomposition strategy (DDS).

Figure 29 - Flow-Chart for selection of the Domain Decomposition Strategy (DDS)

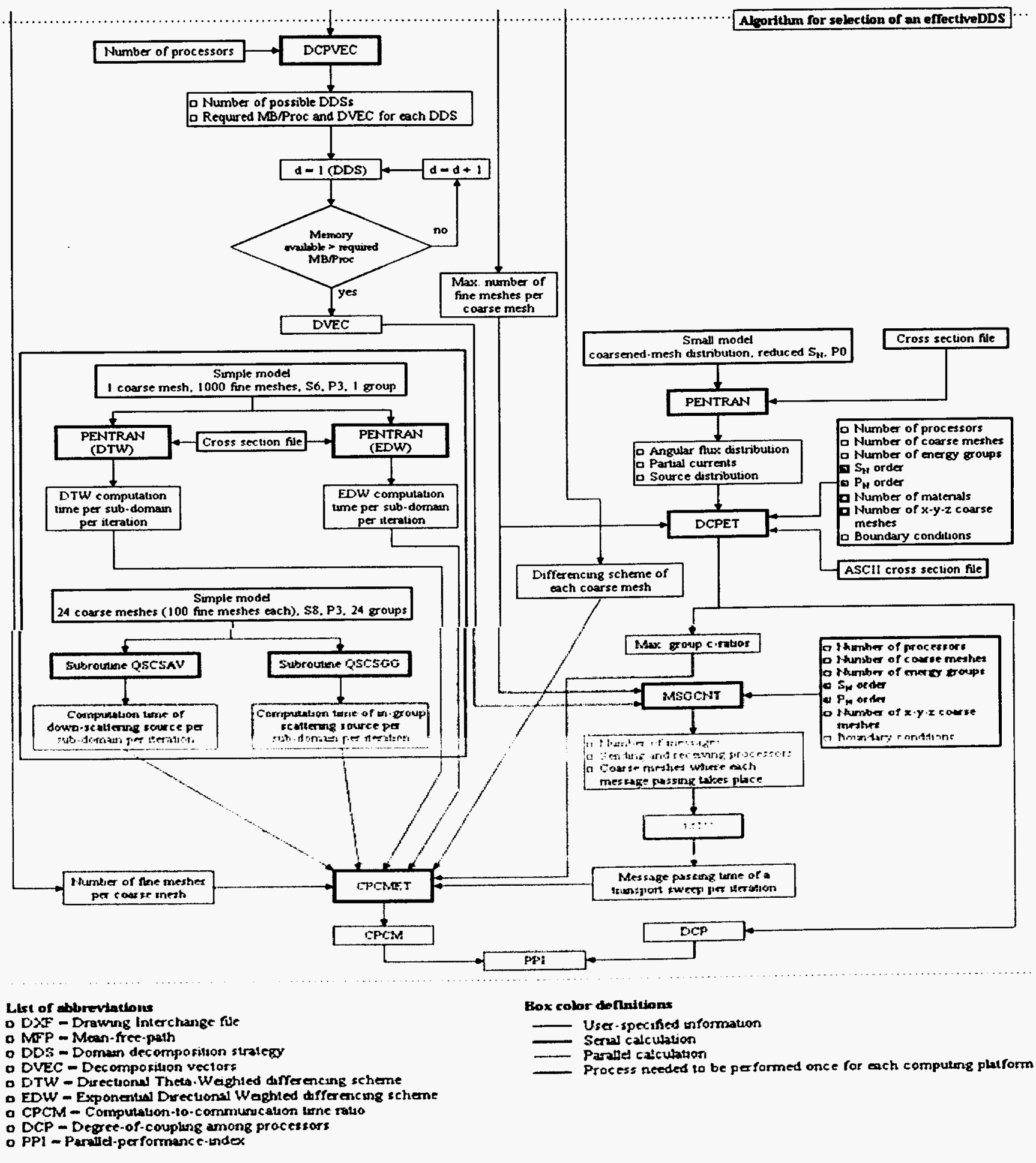


For both test problems, the spatial DDS requires the lowest amount of memory per processor, followed by the angular-spatial and the angular DDSs. This is due to the fact that angular decomposition requires all the spatial arrays for angular fluxes and flux moments on all processors, while the spatial decomposition partitions them among processors. We observe that our predicted CPCM follows a similar trend as the actual CPCM, and that our predicted DCP has a similar behavior as the actual calculation. We define an actual parallel-performance-index (APPI) as the inverse of a transport sweep time. Our predicted PPI, similar to the APPI, indicates that the angular DDS is the most effective DDS. There are other parts of the calculation that are affected by parallel domain decomposition and not included in our algorithm. As a result, the PPI is deviated from the overall APPI, which is estimated based on the inverse of the problem wallclock time. However, we observe a similar trend between the two indexes.

Our domain decomposition selection algorithm accurately predicts [Patchimpattapong and Haghighat, 2003a-c] the effective DDSs for the VENUS-3 and the BWR problems in $\sim 10 \%$ and $\sim 15 \%$ of the actual computation times, respectively. Nonetheless, the majority of the computation time is spent on estimation of the DCP. For these two test problems, the difference in the DCPs does not affect the resulting PPIs, which suggests that the PPI can be simply derived from CPCM. We observe a good agreement between the APPI and the PPI (CPCM). This demonstrates that for these two problems the CPCM is sufficient for obtaining the PPI. As a result, the computation times of our predictive algorithm for the VENUS-3 and the BWR problems are reduced significantly to $\sim 1 \%$ and $4 \%$ of the actual computation times, respectively. For further detail on the expert system and its performance consult Patchimpattapong [2003d] 


\section{Summary and Conclusions}

In this project, we have developed several algorithms and techniques which can improve the efficiency and accuracy of the discrete ordinates $\left(\mathrm{S}_{\mathrm{N}}\right)$ method for solving difficulty particle transport problems in parallel environments. Our studies have resulted in tools which can significantly reduce the necessary time for generation of problem inputs which are prepared based on problem physics and available computer resources.

The Sn methods commonly discretize all the independent variables including space, energy, and angle. This discretization leads to 1 arge s ystems of equations which r equire large amounts of computer m emory and execution time. The m ethod is highly v ersatile, h owever, $s$ uffers from several shortcomings which have been addressed by our group and others.

The major shortcomings of the deterministic $S_{N}$ methods are listed below:

a. Large memory

b. Need for effective differencing schemes which allow for problem physics

c. Slow convergence (iterative $\&$ acceleration techniques)

d. Generation of angular quadrature sets

e. Need for generation of effective multigroup cross section libraries

f. Need for parallel algorithms and codes

g. Need for pre-processing utilities for decision on the following items:

1. Multigroup cross section

2. Spatial and angular mesh

3. Differencing scheme

4. In case of parallel processing, selection of the appropriate Domain

decomposition algorithm depending on the available computing resources

h. Need for post-processing utilities for processing and analysis of results

In this project, we address items b, c, d, g.2-g.4. The remaining items have been addressed by our previous studies.

In Chapter II, we have investigated an adaptive differencing strategy (ADS) which allows for the use of different differencing schemes based on problem physics. Based on the Kobayashi benchmark problems, we have demonstrated that a combination of ADS, Taylor Projection Mesh Coupling (TPMC), and an S20 level-symmetric quadrature order results in a relatively accurate solution with minimal ray-effects in highly absorbing regions.

In Chapter III, we have examined two acceleration techniques: angular multigrid and $\mathrm{SP}_{\mathrm{L}}$ synthetic accelerations. On the angular multigrid, we have concluded that a combination of Nested Iteration (NI), V-Cycle, and PCR (partial current rebalance) yields the lowest number of iterations and the highest value of speedup. For example, for a BWR shielding problem, the number of fine-grid iterations reduces by a factor of $\sim 7.5$ and the CPU time reduces by a factor of $\sim 4.1$.

In Chapter IV, in problems with highly directional sources and/or angular flux distributions (caused by material heterogeneities, and/or containing void or pure absorbers), large number of 
directions and/or biased directions are needed. We have developed new techniques for generation of angular quadrature sets which are capable of creating biased directions for local angular refinement. By simulating a few real-life and benchmark problems, we have demonstrated that the $P_{N}-T_{N}$ algorithm with RAR (regional angular refinement) is the most accurate and efficient approach.

In Chapters V \& VI, we have developed a $\mathrm{SP}_{\mathrm{L}}$ code and investigated a $\mathrm{SP}_{\mathrm{L}}$ synthetic acceleration algorithm, respectively. For this work, we have developed the PENSP $_{L}$ (Parallel Environment Neutral-particle $S_{L}$ ) code which solves a 3-D multigroup $S_{L}$ formulation in a parallel environment. Parallelization is achieved based on moment decomposition. PENSP $_{L}$ has been successfully benchmarked based on criticality and shielding problems. For this implementation, PENSP $_{L}$ uses the Conjugate Gradient (CG) formulation with an Incomplete Cholesky preconditioner.

The PENSP $\mathrm{P}_{\mathrm{L}}$ code has been used as a synthetic acceleration technique for the $\mathrm{Sn}$ method. This new SPL-S $\mathrm{S}_{\mathrm{N}}$ synthetic acceleration technique has resulted in significant reduction in the necessary number of iterations and computation of the Sn method.

In Chapter VII, we have developed an expert system which can create an effective mesh distribution and select the most effective domain decomposition strategy (DDS). For real-life problems, it is demonstrated that the expert system can effectively prepare a mesh distribution and predict the best choice of DDS for the available computing environment.

Future work should focus on further testing of the expert system for different real-life problems and for larger degrees of decomposition, and for testing the $\mathrm{SP}_{\mathrm{L}}-\mathrm{S}_{\mathrm{N}}$ synthetic acceleration for both criticality and shielding problems. We also believe work is needed to develop timedependent algorithms for simulation of reactor kinetics/dynamics, generation of 3-D multigroup homogeneous cross sections for transient conditions and long fuel cycles, parallel algorithms for the generation of sensitivity coefficients for estimation of the calculation uncertainties, and lastly development of algorithms for electron transport problems. 


\section{References}

Alcouffe et al., R. E. (1979) "Computational Efficiency of Numerical Methods for the Multigroup, Discrete Ordinates Neutron Transport Equations: The Slab Case," Nuclear Science and Engineering, 71.

Alcouffe, R. (1977) "Diffusion Synthetic Acceleration Methods for the Diamond Differenced Discrete Ordinates Equation”, Nuclear Science and Engineering, 64, pp. 344-355.

Alcouffe, R. (1991) “A Multigrid Solution of the Three- Dimensional DSA Equation: A Question of Efficiency for Three- Dimensional Transport Equations," Proceedings of Advances in Mathematics, Computations, and Reactor Physics, Pittsburgh.

Alcouffe, R. E. and R.D. O'Dell (1986) "Transport C alculations for Nuclear R eactors," CRC Handbook of Reactor Calculations, Vol. 1, P. 341, Y. Ronen, Ed., CRC Press, Boca Raton.

Barnett, A., J. Morel and D. Harris (1989) "A Multigrid Acceleration Method for the OneDimensional $\mathrm{S}_{\mathrm{N}}$ Equations with Anisotropic Scattering”, Nuclear Science and Engineering, 102.

Brantley, P. S. and E.W. Larsen (2000) "The Simplified $\mathrm{P}_{3}$ Approximation," Nuclear Science and Engineering, 134, pp.1-21.

Briggs, W. (1987) A Multigrid Tutorial, Philadelphia, Pennsylvania, Society for Industrial Applied Mathematics, Lancaster Press.

Carew, J. F. and G. Zamonsky (1999) "Uniform Positive-Weight Quadratures for Discrete Ordinate Transport Calculations," Nuclear Science and Engineering, 131, 199-207.

Carlson B.G. (1971b) "Tables of Equal Weight Quadrature EQN Over the Unit Sphere," Los Alamos Scientific Laboratory Report, LA-4734.

Carlson, B.G. and Lathrop K.D. (1965) "Discrete Ordinates Angular Quadrature of the Neutron Transport Equation," Los Alamos Scientific Laboratory Report, LA-3186.

Gelbard, E. M., J. Davis, and J. Pearson (1959) "Iterative Solutions to the $P_{1}$ and Double- $P_{1}$ Equations," Nuclear Science and Engineering, 5, pp.36-44

Gelbard, E. M.and L. A. Hageman (1969) "The Synthetic Method as Applied to the $\mathrm{S}_{\mathrm{N}}$ Equations," Nuclear Science and Engineering, 37, pp.288-298.

Haghighat, A. (1998) "PENMSH - A 3-D Cartesian Mesh Generator for Sn Codes," Users Manual, Penn State Transport Theory Group, Penn State University, University Park, http://www.hsact.com. 
Haghighat, A. (1999a) "PENINP - A Code for Automatic Preparation of PENTRAN Input file," Users Manual, Penn State Transport Theory Group, Penn State University, University Park, PA, http://www.hsact.com.

Haghighat, A. (1999b) "PENPRL - 3-D Linear Interpolator," Penn State Transport Theory Group, Penn State University, University Park, PA, http://www.hsact.com.

Haghighat A. and G.E. Sjoden (1999c), "Significance of Adaptive Differencing, Variable Grid Density, and TPMC for $\mathrm{S}_{\mathrm{N}}$ Methods" Proceedings of Mathematics and Computation, Reactor Physics and Environmental Analysis in Nuclear Applications, Madrid, Spain.

Haghighat, A. (2000) PENMSH ${ }^{\mathrm{TM}}$ Version 3.3 - A Cartesian-based 3-D Mesh Generator, Users Manual, http://www.hsact.com.

Haghighat, A., G.E. Sjoden, and V.N. Kucukboyaci, (2001) "Effectiveness of PENTRAN's Unique Numerics for Simulation of the Kobayashi Benchmarks," a special issue of the Progress in Nuclear Energy Journal.

Haghighat, A., H. A. Abderrahim, and G.E. Sjoden (2000) "Accuracy and Parallel Performance of PENTRAN Using the VENUS-3 Benchmark Experiment", Reactor Dosimetry, ASTM STP 1398, John G. Williams, et al., Eds., ASTM, West Conshohocken, PA.

Kobayashi, K. (1996) "A Proposal for 3-D Radiation Transport Benchmarks for Simple Geometries with Void Region," Proceedings of the 3-D Deterministic Radiation Transport Computer Programs- Features, Application, and Perspective, 403, OECD report .

Kobayashi, K., N. Sugimura, and Y. Nagaya (1999) "3D Radiation Transport Benchmarks for Simple Geometries with Void Region," Proc. Int. Conf. M\&C'99, Madrid, Spain, pp. 657-666.

Kuckboyaci, V. N. (2001a) "New Angular Multigrid Formulations for Sn Transport Methods and Their Applciaiton to Large 3-D Shielding problems Using Parallel Computing Environment," a PhD Dissertation, Penn State University.

Kucukboyaci, V. and A. Haghighat, G. E. Sjoden and B. Petrovic (2000a) "Modeling of BWR for Neutron and Gamma Fields Using PENTRAN," Reactor Dosimetry, ASTM STP 1398, John G. Williams, et al., Eds., ASTM, West Conshohocken, PA.

Kucukboyaci, V. and A. Haghighat, G. E. Sjoden and B. Petrovic (2000b) "Angular Multigrid Acceleration for Parallel $\mathrm{S}_{\mathrm{N}}$ Method with Application to Shielding Problems," Proceedings of the PHYSOR 2000, Pittsburgh, PA.

Kucukboyaci, V. and A. Haghighat, G. E. Sjoden and B. Petrovic (1999) "A Simplified Angular Multigrid Method to Accelerate Sn Calculations," Transactions of the American Nuclear Society, 14-18, Long Beach, CA. 
Kucukboyaci, V. and A. Haghighat (2001b) "Analysis of Angular V-Cycle Multigrid

Formulation for 3-D Discrete Ordinates Method," Transactions of the American Nuclear Society, Milwaukee, WI, June 17-21.

Lewis, E. E. and G. Palmiotti (1997) "Simplified Spherical Harmonics in the Variational Nodal Method," Nuclear Science and Engineering, 126, pp.48-58.

Longoni, G., A. Haghighat, J. Brown and V. Kucukboyaci (2001a) "Investigation of new quadrature sets for discrete ordinates with application to non-conventional problems", Transactions of the American Nuclear Society, Vol. 84, pp. 224-226, ANS, Milwaukee WI.

Longoni, G. and A. Haghighat (2001b) "Development of new quadrature sets with the Ordinate Splitting technique", Proceedings of the ANS International Meeting on Mathematical Methods for Nuclear Applications, ANS, Salt Lake City UT.

Longoni, G. and A. Haghighat (2002a) "Simulation of a CT-Scan Device with PENTRAN using the new Regional Angular Refinement Technique," Proceedings of the $12^{\text {th }}$ ANS Radiation Protection and Shielding Division Topical Meeting (RPSD 2002), Santa Fe, New Mexico.

Longoni, G. and A. Haghighat (2002b) "Development of the Regional Angular Refinement and its Application to the CT-Scan Device," Transactions of the American Nuclear Society, Vol. 86.

Longoni, G. and A. Haghighat (2002c) "Development and Application of the Regional Angular Refinement Technique and its Application to Non-conventional Problems," Proceedings of PHYSOR 2002, Seoul, Korea, 3-7 October.

Longoni, G., A. Haghighat, and G. Sjoden (2002d) "Development and Application of the Multigroup Simplified $\mathrm{P}_{3}\left(\mathrm{SP}_{3}\right)$ Equations in a Distributed Memory Environment," Proceedings of PHYSOR 2002, Seoul, Korea, 3-7 October.

Longoni, G., and A. Haghighat (2003a) "Development and Applications of the SP $\mathrm{S}_{\mathrm{L}}$ Methodology for a Criticality Eigenvalue Benchmark Problem," Proceeding of the Nuclear Mathematical and Computational Sciences Conference, Gatlinburg, TN, April 6-10.

Longoni., G., A. Haghighat and G. E. Sjoden (2003b) "Implementation and Testing of a Parallel Simplified Spherical Harmonics ( $\mathrm{SP}_{\mathrm{L}}$ ) Algorithm for Reactor Physics Applications," accepted for publication in the proceedings of the International Conference on Supercomputing in Nuclear Applications, Paris, France, 22-24 September.

Miller, W. (1978) "Generalized Rebalance: A Common Framework for Transport Acceleration Methods," Nuclear Science and Engineering, 65, pp. 226-236.

Morel, J. E., J.M. McGhee and E.W. Larsen (1996) “A Three-Dimensional Time-Dependent Unstructured Tetrahedral-Mesh SP $_{\mathbf{N}}$ Method," Nuclear Science and Engineering, 123, pp.319327. 
Morel, J.E. and T.A. Manteuffel (1991) "An Angular Multigrid Acceleration Technique for $\mathrm{S}_{\mathrm{N}}$ Equations with Highly Forward-Peaked Scattering," Nuclear Science and Engineering, 107, pp. $330-342$.

Nowak, P.F., E.W Larsen, and W.R Martin (1988) "A Multigrid Method for $\mathrm{S}_{\mathrm{N}}$ Calculations in x-y Geometry", Transactions of the American Nuclear Society, 56, 291.

Patchimpattapong, A., and A. Haghighat (2000) "Development of an Expert System for Generation of an Effective Mesh Distribution for the $\mathrm{S}_{\mathrm{N}}$ Method," Transactions of the American Nuclear Society, Milwaukee, Wisconsin, June 17-21.

Patchimpattapong, A. and A. Haghighat (2002a) "Developing an Expert System for Preparing an Effective Mesh Distribution for the $\mathrm{S}_{\mathrm{N}}$ Method in the Parallel Environment," Proceedings of the $12^{\text {th }}$ ANS Radiation Protection and Shielding Division Topical Meeting (RPSD 2002), Santa Fe, New Mexico.

Patchimpattapong, A. and A. Haghighat (2002b) "An Expert System for Automatic Mesh Generation for $S_{N}$ Particle Transport Method in Parallel Environment," the 11th International Symposium on REACTOR DOSIMETRY (ISRD 2002), Brussels, Belgium.

Patchimpattapong, A., and A. Haghighat (2003a) "Testing an Expert System for Selection of Mesh Domain Decomposition of Parallel $S_{N}$ Method," Proceeding of the Nuclear Mathematical and Computational Sciences Conference, Gatlinburg, TN, April 6-10.

Patchimpattapong, A. and A. Haghighat (2003b) "An expert system for automatic mesh generation for Sn particle transport simulation in parallel environment," accepted for publication in the proceedings of the International Conference on Supercomputing in Nuclear Applications, Paris, France, 22-24 September.

Patchimpattapong, A., and A. Haghighat (2003c) "Effectiveness of an expert system for selection of parallel Sn domain decomposition strategy," accepted for publication in the Transactions of the American Nuclear Society, San Diego, CA, June 1-5.

Patchimpattapong, A. (2003d) "Development of an Expert System for Automatic Mesh Generation for $\mathrm{S}_{\mathrm{N}}$ Particle Transport Method in Parallel Environment," a $\mathrm{PhD}$ dissertation in Nuclear Engineering, Penn State University.

Pautz, S. D., J.E. Morel, and M. Adams (1999) “An Angular Multigrid Acceleration Method for SN Equations with Highly Forward-Peaked Scattering," Proceedings of Mathematics and Computation, Reactor Physics and Environmental Analysis in Nuclear Applications, Madrid, Spain.

Petrovic, B. and A. Haghighat (1996) "Analysis of Inherent Oscillations in Multidimensional Sn Solutions of the Neutron Transport Equation," Nuclear Science and Engineering, 124. 
Petrovic, B. and A. Haghighat (1998) "New Directional Theta-Weighted $\mathrm{S}_{\mathrm{N}}$ Differencing Scheme and Reduction of Estimated Pressure Vessel Fluence Uncertainty," Reactor Dosimetry, 746-753, edited by H. A. Abderrahim, P. D'hondt, and B. Osmera, World Scientific Publishing Company.

Petrovic, B., A. Haghighat, T. Congedo, and A. Dulloo (1999) "Hybrid Forward Monte CarloAdjoint Sn Methodology for Simulation of PGNAA Systems," Proc. Int. Conf. M\&C'99, Madrid, Spain, pp. 1016-1025.

Reed, W. H. (1971) "The Effectiveness of Acceleration Techniques for Iterative Methods in Transport Theory," Nuclear Science and Engineering, 45, pp.245-254.

Rhoades, W. (1981) "Improvements in Discrete Ordinates Acceleration", Transactions of the American Nuclear Society, 39, 753-755.

Rhoades, W. A. and W.W. Engle, Jr. (1977) "A New Weighted-Difference Formulation for Discrete Ordinates Calculation," Transactions of the American Nuclear Society, 27, 776.

Sjoden, G. and A. Haghighat (1996a) "A New Adaptive Differencing Strategy in PENTRANTM 3-D Parallel Code," Transactions of the American Nuclear Society, 75, 148.

Sjoden, G. and A. Haghighat (1996b) "A Simplified Multigrid Acceleration in the PENTRAN 3D Parallel $\mathrm{S}_{\mathrm{N}}$ code," Transactions of the American Nuclear Society, 75, 152.

Sjoden, G. and A. Haghighat (1997) "PENTRAN- Parallel Environment Neutral- particle TRANsport in 3-D Cartesian Geometry," Proceedings of Joint International Conference on Mathematical Models and Supercomputing for Nuclear Applications, Saratoga Springs, New York.

Sjoden, G. E. (1999) "PENDATA - Data Post-Processor for PENTRAN Outputs," Penn State Transport Theory Group, Penn State University, University Park, PA, http://www.hsact.com.

Sjoden, G. E. and A. Haghighat (1996a) "A New Adaptive Differencing Strategy in PENTRAN 3-D Parallel Code," Transactions of the American Nuclear Society, 75, 148.

Sjoden, G. E. and A. Haghighat (1996b) "Taylor Projection Mesh Coupling Between 3-D Discontinuous Grids for Sn," Transactions of the American Nuclear Society, 74, 178-179.

Sjoden, G. E. and A. Haghighat (1997a) "The Exponential Directional Weighted (EDW) Sn Differencing Scheme in 3-D Cartesian Geometry," Proceedings of the 1997 Joint International Conference on Mathematical Methods and Supercomputing for Nuclear Applications, Vol. 2, 1267, Saratoga Springs, NY.

Sjoden, G. E. and A. Haghighat (1997b) "PENTRAN - A 3-D Cartesian Parallel Sn Code with Angular, Energy, and Spatial Decomposition," Proceedings of the 1997 Joint International 
Conference on Mathematical Methods and Supercomputing for Nuclear Applications, Vol. 1, 553, Saratoga Springs, NY.

Sjoden, G. E., R. N. Gilchrist, D. L. Hall and C. A. Nusser (2000) "Modeling a Radiographic XRay Imaging Facility with the PENTRAN Parallel $S_{N}$ Code," proceedings of the PHYSOR 2000, Pittsburgh, PA.

Takeda, T. and H. Ikeda (1991) NEACRP-L-330. 